\title{
PRIVILEGED COMMUNICATION
}

\section{NRF1 Association with AUTS2-Polycomb Mediates Specific Gene Activation in the Brain}

Sanxiong Liu ${ }^{1,2}$, Kimberly A Aldinger ${ }^{3}$, Chi Vicky Cheng ${ }^{3}$, Takae Kiyama ${ }^{4,5}$, Mitali Dave ${ }^{3}$, Hanna K. McNamara ${ }^{3}$, Stefano G Caraffi ${ }^{6}$, Ivan Ivanovski ${ }^{6}$, Edoardo Errichiello ${ }^{7}$, Christiane Zweier $^{8}$, Orsetta Zuffardi ${ }^{7}$, Michael Schneider ${ }^{9}$, Antigone S. Papavasiliou ${ }^{10}$, M. Scott Perry ${ }^{11}$, Megan T Cho $^{12}$, Astrid Weber ${ }^{13}$, Andrew Swale ${ }^{13}$, Tudor C. Badea ${ }^{14}$, Chai-An Mao ${ }^{4,5}$, Livia Garavelli ${ }^{15}$, William B Dobyns ${ }^{3,16}$, Danny Reinberg ${ }^{1,2,17, *}$

${ }^{1}$ Department of Biochemistry and Molecular Pharmacology, New York University Langone School of Medicine, New York, NY 10016, USA

${ }^{2}$ Howard Hughes Medical Institute, Chevy Chase, MD 20815, USA

${ }^{3}$ Center for Integrative Brain Research, Seattle Children's Research Institute, Seattle, WA

${ }^{4}$ Ruiz Department of Ophthalmology and Visual Science, McGovern Medical School at The University of Texas Health Science Center at Houston (UTHealth), Houston, TX 77030, USA

${ }^{5}$ National Institutes of Health-National Eye Institute to CAM (EY024376)

${ }^{6}$ Struttura Semplice Dipartimentale di Genetica Medica, Azienda USL-IRCCS di Reggio Emilia,

Reggio Emilia, Italy

${ }^{7}$ Dipartimento di Medicina Molecolare, Università di Pavia, Pavia, Italy

${ }^{8}$ Institute of Human Genetics, Friedrich-Alexander-Universität Erlangen-Nürnberg (FAU),

Schwabachanlage 10, 91054, Erlangen, Germany

${ }^{9}$ Carle Physicians Group, Section of Neurology, St. Christopher's Hospital for Children, Urbana, IL

${ }^{10}$ Neurology, IASO Children's Hospital, Athens Greece

${ }^{11}$ Comprehensive Epilepsy Program, Jane and John Justin Neuroscience Center, Cook Children's

Medical Center, Fort Worth, TX 76104

${ }^{12}$ GeneDx, Inc

${ }^{13}$ Liverpool Women's Hospital UK

${ }^{14}$ National Eye Institute, NIH, Bethesda, MD 20892, USA

${ }^{15}$ Struttura Semplice Dipartimentale di Genetica Medica, Azienda USL-IRCCS di Reggio Emilia,

Reggio Emilia, Italy

${ }^{16}$ Department of Pediatrics (Genetic Medicine), University of Washington, Seattle, WA

${ }^{17}$ Lead Contact

*Correspondence:danny.reinberg@nyumc.org 


\section{SUMMARY}

The heterogeneous complexes comprising the family of Polycomb Repressive Complex 1 (PRC1) are instrumental to establishing facultative heterochromatin that is repressive to transcription. Yet, two PRC1 species, PRC1.3 and PRC1.5, are known to comprise novel components, AUTS2, $\mathrm{P} 300$, and CK2 that convert this repressive function to that of transcription activation. Here, we report that patients harboring mutations in the HX repeat domain of AUTS2 exhibit defects in AUTS2 and P300 interaction as well as a developmental disorder reflective of Rubinstein-Taybi syndrome, which is mostly associated with a heterozygous pathogenic variant in $C R E B B P / E P 300$. As well, the absence of AUTS2 gives rise to a mis-regulation of a subset of developmental genes and curtails motor neuron differentiation from embryonic stem cells in the context of a well-defined system. Moreover, the transcription factor, Nuclear Respiratory Factor 1 (NRF1) exhibits a novel and integral role in this aspect of the neurodevelopmental process, being required for PRC1.3 recruitment to chromatin. 


\section{INTRODUCTION}

The establishment, maintenance, inheritance and regulated dissolution of facultative heterochromatin(Trojer and Reinberg, 2007) is paramount to the developmental processes that give rise to the distinct cellular identities comprising multi-cellular organisms. The Polycomb group (PCG) of proteins are required for the formation and integrity of facultative heterochromatin as a function of the presence of intracellular signals that occur during development, as well as for the maintenance of adult tissue-specific gene expression profiles(Bonasio et al., 2010; Margueron and Reinberg, 2011; Di Croce and Helin, 2013). Indeed, our findings demonstrate that in contrast to chromatin domains engaged in active transcription, it is the repertoire of repressive chromatin domains that are conveyed to daughter cells upon DNA replication, thereby maintaining cellular identity(Escobar et al., 2019, 2021).

Two multi-subunit complexes, Polycomb Repressive Complex-1 and -2 (PRC1 and PRC2, respectively) comprise a defined subset of PcG proteins(Schuettengruber et al., 2017), and act in concert to establish facultative heterochromatin. PRC2 comprises the sole activity that catalyzes mono-, di, and tri-methylation of histone H3 at lysine 27 (H3K27me1, -me2, -me3, respectively), with chromatin domains comprising $\mathrm{H} 3 \mathrm{~K} 27 \mathrm{me} 2 / \mathrm{me} 3$ forming the platform for chromatin compaction(Lau et al., 2017; Oksuz et al., 2018; Yu et al., 2019; Kim and Kingston, 2020). PRC1 complexes comprise other subsets of the PCG protein family. We and others previously characterized at least six heterogeneous PRC1 subcomplexes, each of which comprise one of the six Polycomb Group Ring Finger (PCGF1-6) components and RING1A and/or RING1B(Gao et al., 2012; Tavares et al., 2012; Hauri et al., 2016). This heterogenicity of the PRC1 complexes resulted in their classification into two major PRC1 subcomplexes. Canonical PRC1 (CPRC1) comprise one of several CBX components that bind to chromatin containing nucleosomes decorated with H3K27me3 catalyzed by PRC2, thereby resulting in chromatin compaction and thus, transcription repression(Min et al., 2003; Francis et al., 2004; Gao et al., 2012; Lau et al., 2017; Kim and Kingston, 2020). The non-canonical set of PRC1 (ncPRC1) comprise either RYBP or YAF2 that stimulate the catalysis of H2A mono-ubiquitinated lysine 119 (H2AK119ub1) through the common PRC1 subunit, RING1A/B. Of note, incorporation of CBX or either RYBP or YAF2 into PRC1 is competitive such that CPRC1 is devoid of RYBP and YAF2 and ncPRC1 is devoid of CBX(Wang et al., 2010; Gao et al., 2012). The joint effect of PRC2/ncPRC1 arises from a PRC2 accessory partner, Jarid2, which stimulates PRC2 activity(Li et al., 2010; Pasini et al., 2010). Jarid2 reportedly interacts with the ncPRC1-mediated product, H2AK119ub1(Kasinath et al., 2021). Interestingly, while this joint recruitment of PRC2 with either CPRC1 or ncPRC1 is distinct, both of these versions of PRC1 are found in proximity at select genome-wide regions(Gao et al., 2012; Scelfo et al., 2019). Most importantly, this PRC2/PRC1 network is paramount to fostering the appropriate profiles of facultative heterochromatin evident during development and in adulthood(Margueron and Reinberg, 2011; Aloia et al., 2013; Schuettengruber et al., 2017).

Our previous characterization of PRC1 complexes also revealed that a subset of ncPRC1 that comprise either PCGF3 (ncPRC1.3) or PCGF5 (ncPRC1.5) unexpectedly contained three non-PcG 
proteins: AUTS2, CK2 and P300(Gao et al., 2012, 2014). Remarkably, we found that these proteins hijack the normally repressive ncPRC1 complex and convert it into a transcriptional activator(Gao et al., 2014). Indeed, CK2 mediates the phosphorylation of the RING1A/B subunit of PRC1, thereby thwarting its catalysis of H2AK119ub1. Moreover, AUTS2 interacts with PCGF3/-5 and most importantly, recruits P300/CBP, a known transcriptional co-activator possessing histone acetyltransferase activity(Bannister and Kouzarides, 1996; Ogryzko et al., 1996). The presence of AUTS2, P300/CBP and CK2 within two ncPRC1 complexes (PRC1.3/1.5) converts the function of these complexes into that of transcription activation, rather than repressive activity(Gao et al., 2014). These findings point to AUTS2 having a profound impact on gene expression in the context of defined aspects of development. Indeed, AUTS2-ncPRC1.3 is important during development of the central nervous system (CNS) as well as in the postdevelopmental stage (see below). In contrast, AUTS2-ncPRC1.5 appears to function in the establishment and maintenance of other lineages of differentiation (our unpublished results). Notably, ncPRC1.3/-1.5 comprises another non-PCG protein, FBRSL1, which shares a highly similar protein sequence with AUTS2(Gao et al., 2012). Interestingly, AUTS2 and FBRSL1 bind competitively to the PCGF subunit of ncPRC1.3/-1.5(Gao et al., 2014).

The gene encoding AUTS2 was designated as such based on the identification of its translocation in a pair of monozygotic twins that were diagnosed with autism(Sultana et al., 2002); yet its role in Autism Spectrum Disorders (ASD) is still putative. Nonetheless, the role of AUTS2 in neurodevelopment has been more widely established through the identification of variants in AUTS2 that are associated with variable intellectual disability (ID), outgoing social behavior, mild microcephaly, and selected co-morbid features of autism that include obsessivecompulsive behavior(Beunders et al., 2013; Oksenberg and Ahituv, 2013; Hori and Hoshino, 2017). Various reports indicate the association of AUTS2 variants with other pronounced neurological diseases, including epilepsy, bipolar disorder, attention deficit hyperactive disorder, and alcohol dependency(Hattori et al., 2009; Mefford et al., 2010; Elia et al., 2010; Kapoor et al., 2013). Notably, a thorough and informative report analyzed AUTS2 binding to chromatin genome-wide in mouse E16.5 forebrain, finding that AUTS2 is bound to gene promoters and enhancers whose function appears to be important during neurodevelopment(Oksenberg et al., 2014), and pointing to its substantive role in activating genes required for appropriate CNS development and function.

Given that AUTS2 interacts with P300, converting nCPRC1.3 into a transcriptional activator, it is notable that human heterozygous pathogenic variants in EP300 or CREBBP (CREB Binding Protein/CBP) are associated with Rubinstein-Taybi syndrome (RSTS), a neurodevelopmental disorder characterized by distinctive facial features, broad and angulated thumbs, short stature, and intellectual disability(Stevens, 1993; Ajmone et al., 2018). Here, we report heterozygous de novo variants in AUTS2 in patients who exhibit a severe phenotype overlapping that of RSTS. Notably, these new AUTS2 variants are defective in P300/CBP interaction, underscoring the biological relevancy of P300/CBP incorporation into AUTS2-ncPRC1.3 with respect to appropriate neurodevelopment and brain function in human. 
We further extended our studies to understand the means by which AUTS2-ncPRC1.3 accesses specific chromatin sites in the brain and identified the transcription factor, Nuclear Respiratory Factor 1 (NRF1), as being instrumental to this process. Previous studies implicated NRF1 in mitochondrial biogenesis(Scarpulla, 2011) and appropriate development of the retina(Hsiao et al., 2013; Kiyama et al., 2018). Here we demonstrate that NRF1 mediates AUTS2-ncPRC1.3 recruitment to a subset of neurodevelopmental genes during differentiation of mouse embryonic stem cells to motor neurons, as well as in the mouse brain during early development. These findings expose a novel and key role for NRF1 in facilitating appropriate AUTS2-ncPRC1.3-mediated activation of genes involved in neurodevelopment as a consequence of AUTS2-P300 interaction. 


\section{RESULTS}

\section{ncPRC1.3 occupies active genes during early development in the mouse brain}

The Auts2 gene encodes 2 major transcript isoforms in the mouse brain(Hori et al., 2014). The full-length (FL) mouse Auts2 transcript (Auts2-1, Figure 1A) includes 19 exons, and produces the long isoform of AUTS2 (AUTS2_L, 1-1261 aa, Figure 1B). Another Auts2 transcript (Auts2-2, Figure $1 \mathrm{~A}$ ) arises from a transcriptional start site near exon 7 and comprises a translational start site in the middle of exon 8 that gives rise to the short form of AUTS2 protein (AUTS2_S, 4581261 aa, Figure 1B)(Hori et al., 2014). Both AUTS2 isoforms contain a PY motif and an HX repeat that in the case of AUTS2-L is located between two proline rich regions (PR1 and PR2) in the Nterminus (Figure 1B). The PY motif is a potential WW-domain-binding region present in various activating transcription factors(Sultana et al., 2002). The HX repeat (aa 525-542) comprises alternating $\mathrm{HQ}(\times 6)$ or $\mathrm{HT}(\mathrm{x} 3)$ residues, mutations of which in two other genes (ATN1 and $R E R E$ ), are related to neurodevelopmental disorders(Jordan et al., 2018; Palmer et al., 2019).

To investigate the role of AUTS2 specifically in the context of its associated ncPRC1 complex in brain development, we examined its expression and that of the core PRC1 components in the mouse brain throughout early development. Western blot analysis in whole mouse brain lysates using an antibody against the AUTS2 C-terminus [1160-1259 amino acids of human AUTS2 protein(Gao et al., 2014)], showed expression of both FL AUTS2 protein (approximately $170 \mathrm{kDa}$ ) and its shorter form (approximately $95 \mathrm{kDa}$ ) with the latter being predominant (Figure 1C). The expression of both isoforms gradually decreased throughout early development (Figure 1C). Accordingly, both Ring1B and PCGF3 expression subsided dramatically from postnatal day 5 (P5) (Figure 1C).

As AUTS2 is incorporated into both ncPRC1.3 comprising PCGF3 and ncPRC1.5 comprising PCGF5 in 293 T-REx cells(Gao et al., 2012), we examined the expression of Pcgf3 and Pcgf5 from whole brain lysates at postnatal day 1 . Interestingly, RNA-Seq data revealed that Pcgf3, but not Pcgf5, was predominantly expressed in the mouse brain (Figure 1D). Mass spectrometry (MS) analysis following co-immunoprecipitation (co-IP) experiments from brain lysate, using AUTS2 antibody recovered considerably more peptides from Pcgf3 than from Pcgf5 (Figure 1E). As previously reported(Gao et al., 2014), Two other components comprising ncPRC1.3, Ring1A/B and casein kinase 2 (CK2), were also observed (Figure 1E). Importantly, brain-specific conditional knockout of $P c g f 3$ (Pcgf3 $3^{\text {loxp/loxp }}:$ Nes $^{\text {Cre }}$ ) caused lethality (data not shown), suggesting a critical role for AUTS2-ncPRC1.3 during early brain development.

To better understand how AUTS2-ncPRC1.3 is involved in transcriptional regulation, we next characterized the genomic localization of AUTS2, P300, and the ncPRC1.3 components: RING1B, RYBP and PCGF3, by ChIP followed by deep sequencing (ChIP-seq) in whole brain lysate at postnatal day one. Consistent with our previously published ChIP-seq data in mouse brain, AUTS2 associated with ncPRC1.3 components including P300 in the promoter of active genes that were devoid of histone post-translational modifications (hPTMs) associated with transcription repression, and instead exhibited strong signals for hPTMs associated with active 
transcription, e.g. H3K27ac and H3K4me3 (Figure 1F). This finding was corroborated by a genome-wide analysis (Figure $1 \mathrm{G}$ ), and together provide strong evidence that AUTS2-ncPRC1.3 is involved in active transcription in the mouse brain. $\mathrm{GO}$ analysis revealed that terms related to RNA processing and neuronal development were enriched in genes located in AUTS2-bound regions (Figure $\mathrm{S} 1$ ).

\section{Patients with mutations in the AUTS2 HX repeat share features with Rubinstein-Taybi syndrome}

We previously reported that a truncated form of AUTS2 protein (404 to 913 aa) was sufficient to mediate transcriptional activation through its recruitment of P300(Gao et al., 2014). Yet, the AUTS2 residues key to its interaction with P300 and the physiological relevance of AUTS2-P300 interaction during brain development remained largely unexplored. As part of an ongoing effort to identify genetic variants associated with developmental brain disorders(Aldinger et al., 2019), we detected a novel de novo missense variant in AUTS2 in a boy with multiple congenital anomalies and a proposed diagnosis of RSTS. His phenotype was more severe than the syndrome previously reported among individuals with heterozygous AUTS2 deletions(Beunders et al., 2013). To investigate the clinical phenotype associated with AUTS2 mutations, we identified 6 additional individuals with de novo intragenic variants that were clustered in exon 9 of the AUTS2 coding region (NM_015570.2) (Figures 2A, S2 and Table S1). All 7 individuals displayed dysmorphic features and feeding difficulties in infancy, and most had moderate to severe intellectual disability and hypotonia (Table S1). Importantly, 5 of these individuals harbored mutations within a short histidine-rich HX repeat motif (aa 525-542) that comprises alternating $\mathrm{HQ}(\mathrm{x} 6)$ or $\mathrm{HT}(\mathrm{x} 3)$ residues, all of whom had severe phenotypes (Table S1). Our original proband (LR05-007) had a missense mutation, p.Thr534Pro, while the remaining four individuals (LR15-003, LR18-404, LR19-314, LR19-506) had an identical recurrent small deletion within exon 9: p.His535_Thr542del (Figure 2A). Notably, pathogenic variants in the HX repeat of two other genes [ATN1(Palmer et al., 2019) and RERE(Jordan et al., 2018)] are also associated with neurodevelopmental disorders (Figure 2B).

All 5 patients with mutations in the HX repeat of AUTS2 had a dysmorphic facial appearance dominated by features seen in RSTS, although less severe than classic RSTS (Table S1). RSTS is a complex multiple congenital anomaly syndrome characterized by short stature, distinctive facial features, and varying degrees of intellectual disability(RUBINSTEIN and TAYBI, 1963; Wiley et al., 2003). In most individuals, RSTS is associated with mutations in genes (CREBBP, EP300) encoding the CREB binding protein (CBP) or P300, or a microdeletion of $16 \mathrm{p} 13.3$ that includes CREBBP(Stevens, 1993). A clinical diagnosis of RSTS was suggested for 2 of the 5 individuals prior to genetic testing (Table S1). The two other patients with mutations outside of the HX repeat domain did not exhibit a phenotype overlapping that of RSTS (Table S1), but display other neurological defects. Moreover, neuroimaging studies from 4 of the 5 patients with mutations in the HX repeat showed hypoplasia of the corpus callosum $(n=3)$, cerebellar hypoplasia and small posterior fossa $(n=4)$, and Chiari malformation type $1(n=1)$ (Figure 2C and Table S1). Such brain malformations are also reported in individuals with classic RSTS(Cantani 
and Gagliesi, 1998; Ajmone et al., 2018). These findings led us to hypothesize that the HX repeat in AUTS2 coordinates with CBP/P300 in regulating proper gene expression in the brain.

\section{The HX repeat in AUTS2 interacts with $\mathrm{P} 300$, crucial for activating transcription}

Given that mutations in both the AUTS2 HX repeat and CBP/P300 are associated with RSTS, we next sought to determine whether the RSTS phenotype observed could be due to disrupted interactions between AUTS2 and CBP/P300. To this end, we initially expressed Flag-tagged AUTS2, either wild-type (WT) or patient-derived mutant forms including two variants with in the $\mathrm{HX}$ repeat (T534P and 535-542aa del) and one outside this region (P517L within the PY motif) in 293 T-REx cells (Figures 3A and 2A). Strikingly, co-IP experiments using Flag-WT or Flag-mutant AUTS2 revealed that both mutations within the HX repeat (T534P and 535-542aa del), but not P517L, disrupted interaction with P300 (Figure 3B). This finding is in accordance with the clinical diagnosis of RSTS for patients harboring mutations with in the HX repeat, but not the P517L mutation (Table S1). Of note, WT and all AUTS2 variants interacted stably with Ring1B (Figure $3 \mathrm{~B}$ ), suggesting that AUTS2 regions outside the PY motif and HX repeat could mediate AUTS2 incorporation into the ncPRC1 complex. Importantly, reciprocal co-IP experiments performed against endogenous P300 confirmed that its interaction with AUTS2 was disrupted when AUTS2 was mutant in its HX repeat (Figure $3 \mathrm{C}$ ). Similar results were observed using a more relevant system: cells undergoing differentiation into motor neurons (see below).

Considering that P300 is required for AUTS2-mediated transcriptional activation(Gao et al., 2014), we next wondered whether AUTS2 mutants in the HX repeat lose the ability to activate transcription due to their disrupted interaction with P300. To this end, GAL4-AUTS2, either WT or mutant in the HX repeat, or GAL4 alone were inducibly expressed in 293 T-REx cells containing an integrated luciferase reporter with a UAS comprising five consecutive GAL4 DNA binding sites (Figure 3D). Importantly, we found a severe defect in doxycycline-mediated induction of luciferase activity in the case of the GAL4-AUTS2 mutants compared to WT (Figure 3E).

Finally, to confirm the critical role of the HX repeat domain in mediating interaction between AUTS2 and P300 in a more relevant system (in vitro neuronal differentiation), we generated mouse embryonic stem cells ( $\mathrm{mESC}$ ) genetically modified to harbor one of two mutations in the AUTS2 HX repeat domain at the endogenous locus (T534P or a 535-542 aa deletion, Figures S3A and $\mathrm{S} 3 \mathrm{~B}$, respectively), which we could then differentiate toward the neuronal lineage (see Figure 5A for more details). Introduction of either mutation at the endogenous locus did not change the expression level of AUTS2 (Figure S3C). Consistent with our observations in 293T cells, patient-derived mutations in the AUTS2 HX repeat domain disrupted its interaction with P300 but not its incorporation into the ncPRC1 complex (Figures $3 \mathrm{~F}$ and 3G). Altogether, these results establish that the AUTS2 HX repeat (aa 525-542) engages in P300 interaction, pointing to the critical role of this minimal region in AUTS2-mediated transcriptional activation and corroborating the consequences of the HX mutation in Rubinstein-Taybi syndrome (RSTS). 
To identify the factor(s) involved in the key process by which AUTS2 is recruited to chromatin, we first determined the motifs of transcription factors (TFS) enriched in AUTS2-bound sites in the mouse brain and identified an overrepresentation for that of Nuclear Respiratory Factor 1 (NRF1) (Figure 4A). NRF1 is a TF known for its role in mitochondrial biogenesis(Scarpulla, 2011), and binds to GC-rich DNA elements in promoters of many mitochondrial biogenesis-related genes(Evans and Scarpulla, 1990; Gleyzer et al., 2005). As well, the protein is associated with the regulation of neurite outgrowth(Chang et al., 2005; Tong et al., 2013) and exhibits essential roles in retinal development(Hsiao et al., 2013; Kiyama et al., 2018), yet its function and regulation in the CNS is largely unknown. To validate our computational prediction, we performed ChIP-seq for NRF1 using 2 different antibodies and lysates from whole mouse brain isolated at postnatal day one and ascertained that the majority of AUTS2 peaks (1545 of 2005 peaks in total) were associated with genomic sites bound by NRF1 (Figures 4B and 4C).

Based on this high overlap between chromatin-bound AUTS2 and NRF1, we next tested the possibility that NRF1 might physically interact with AUTS2, thereby contributing to their colocalization. Reciprocal co-IP assays using endogenous proteins revealed that indeed, NRF1 physically associates with AUTS2 in the mouse brain (Figure 4D). Notably, the core component of AUTS2-ncPRC1.3, PCGF3, also co-immunoprecipitated with NRF1 indicating that AUTS2 interacts with NRF1 within the context of the ncPRC1.3 complex in mouse brain (Figure 4D). Moreover, the expression of NRF1 recapitulated the pattern of AUTS2-ncPRC1.3 and CBP/P300 expression during early brain development (Figures $4 \mathrm{E}$ and $1 \mathrm{C}$ ). These data strongly suggest that NRF1 contributes to the recruitment of AUTS2 and its associated ncPRC1 complex, although it is also clear that both have independent targets, likely due to their additional functions and partnership with other factors.

AUTS2 and NRF1 colocalize with ncPRC1.3 at actively transcribed loci in cells induced to differentiate to motor neurons

To understand the underlying mechanism that coordinates both the chromatin binding of and the regulation of transcription by AUTS2-ncPRC1.3 and NRF1, we utilized the system by which differentiated motor neurons (MN) are attained in vitro(Wichterle et al., 2002; Mazzoni et al., 2013; Narendra et al., 2015). Under these conditions, the expression of both AUTS2 and PCGF3 was significantly up-regulated in MN, while that of NRF1 decreased to approximately half of its expression in $\mathrm{mESC}$ at both the protein (Figure 5A) and RNA levels (Figure S4A). In contrast, the overall level of PRC1 complex as reflected by that of RING1B was down-regulated (Figures 5A and S4A), consistent with its essential role in maintaining mESC identity(Endoh et al., 2008). To complement our previously published ChIP-seq data for RNA polymerase II (RNAPII) in both mESC and MN(Narendra et al., 2015; LeRoy et al., 2019), we performed similar ChIP-seq for AUTS2 and NRF1. The majority of regions that gained AUTS2 binding upon MN differentiation, also accumulated NRF1 binding (Figure 5B). Importantly, these regions became actively transcribed during differentiation as evidenced by an increase in RNAPII binding (Figure 5B). 
To examine whether AUTS2 cooperates with ncPRC1.3 for active transcription in MN, we next analyzed the genome-wide distribution of a set of hPTMs (H2AK119ub, H3K27me3, H3K27ac and H3K4me3), and the core PRC1 subunits, RING1B and RYBP. Consistent with previous studies in other systems(Kloet et al., 2016; Cohen et al., 2018; Loubiere et al., 2020), k-means clustering revealed three discrete classes of Ring1B-bound regions in $\mathrm{MN}$ (Figure $5 \mathrm{C}$ ). In cluster 1, we observed strong and broad ChIP-seq signals for RING1B, H2AK119ub, and H3K27me3, and the absence of signals for H3K27ac, H3K4me3, RNAPII and the noncanonical PRC1 component, RYBP/YAF2 (RYBP antibodies do not distinguish between RYBP and YAF2), suggesting that these regions are co-repressed by PRC2 and canonical PRC1. The second cluster exhibited lower levels of H2AK119ub and H3K27me3, and increased levels of H3K27ac and H3K4me3, these last two mostly abundant at the peak center, suggesting that these Ring1B-bound regions featured bivalency(Bernstein et al., 2006; Voigt et al., 2013). The third cluster exhibited both H3K27acand H3K4me3-marked active regions enriched for developmental GO terms (Figure S4B). Surprisingly, cluster 3 exhibited elevated RYBP/YAF2 levels. Importantly, both AUTS2 and NRF1 binding were specifically enriched in cluster 3 (Figure 5C). Accordingly, genes flanking cluster 3 regions were expressed at levels significantly higher than those in clusters 1 and 2 (Figure 5D). These data strongly suggest that the transcription factor NRF1 associates with AUTS2-ncPRC1 to facilitate active transcription in motor neurons.

\section{NRF1 directs AUTS2-ncPRC1 chromatin binding}

To ascertain whether AUTS2 binding to chromatin is dependent upon NRF1 binding or vice versa, we first performed ChIP-seq analysis for the presence of AUTS2 or NRF1 as a function of depleting either NRF1 or AUTS2, respectively. Exon 9 of the Auts2 gene was targeted by CRISPRCas9 to remove both the long and short forms of the protein (Figures S4C-E, Table S2) and exon 4 of the Nrf1 gene was targeted by CRISPR-Cas9 to remove NRF1 protein (Figures S4F-H, Table S2) in mESC from which MN were then derived. As a consequence of NRF1 depletion, most AUTS2-binding events were decreased in MN (Figures 5E and S5A). However, NRF1 ChIP-seq signals remained largely unaltered upon AUTS2 depletion (Figures 5F and S5B), demonstrating that binding of AUTS2 is NRF1-dependent, but NRF1 binding to chromatin is independent of AUTS2.

We next probed how NRF1-directed AUTS2 binding might modulate ncPRC1.3-associated active transcription. Under NRF1 depleted conditions, we performed ChIP-seq for Ring1B and a set of hPTMs (H2AK119ub, H3K27me3 and H3K27ac) followed by k-means clustering analysis. Remarkably, we did not recover the cluster of Ring1B-bound active regions (labeled by H3K27ac, but not by H3K27me3 or H2AK119ub), relative to the control (Figures 5G and 5C), implying that ncPRC1.3 was no longer associated with active transcription in the absence of NRF1. Collectively, these data demonstrate a pivotal role for NRF1 in facilitating ncPRC1.3associated active transcription by directing AUTS2 binding to chromatin in motor neurons.

The absence of AUTS2 or NRF1 leads to a defect in PNP to MN differentiation 
Despite much evidence indicating that mutations in the AUTS2 gene are associated with multiple neurodevelopmental disorders, including ASD(Oksenberg and Ahituv, 2013), and the essential role(s) of NRF1 in retinal development(Hsiao et al., 2013; Kiyama et al., 2018), that AUTS2 and NRF1 might coordinately regulate the process of neuronal differentiation was not previously recognized. To characterize gene expression changes during the transition from multipotent, posterior neural progenitors (PNPs) to terminally differentiated MNs upon ablation of AUTS2 or NRF1, we performed single-cell RNA sequencing (scRNA-seq) on MN differentiated from mESC either WT, or having a knockout (KO) of Auts2 (Auts2-KO) or Nrf1 (Nrf1-KO) (see Methods). We chose the newly developed Smart-seq3 technique(HagemannJensen, 2020; Hagemann-Jensen et al., 2020), an improved version of Smart-seq2 with a 5'unique molecular identifier RNA counting strategy and a much higher sensitivity that detects thousands more transcripts per cell. We obtained 632 high-quality (cells with $>3000$ detected genes) single-cell transcriptomes from all samples (WT, 228 cells; Auts2-KO, 221 cells; Nrf1-KO, 183 cells) for in-depth analyses (Figures 6A and S6A). To identify major cell types, we performed unsupervised clustering on a graph-based representation of the cellular gene expression profiles and 5 major clusters were visualized in a uniform manifold approximation and projection (UMAP) embedding(Butler et al., 2018; Becht et al., 2019) and represented by colorcoded dashed-line circles (Figure 6A). Clusters were annotated according to known markers and previously established lineage information(Wichterle et al., 2002; Briggs et al., 2017), i.e., posterior neural progenitor (PNP) expressing Sox3, posterior and ventral neural progenitor (PVNP) expressing Hoxd4, newborn motor neuron (NMN) expressing Neurog2, and motor neuron (MN) expressing $\mathrm{Mnx} 1$ and Chat (Figures S6B and S6C). Importantly, cells from all samples were clustered by cell type identity rather than sample identity (Figure 6A), indicating little or no batch effect. Notably, Auts2 and Nrf1 were highly expressed in all cell types (Figure $\mathrm{S6C}$ ), suggesting their involvement in all of the different stages of differentiation.

To pursue the potential functional requirement of AUTS2 and NRF1 for proper MN differentiation, we first compared the percentage of $M N$ (including NMN and MN) and PNP under WT, Auts2-KO and Nrf1-KO conditions. The percentage of PNP was retained at a much higher level in both $\mathrm{KO}$ conditions (52\% in WT, 68\% and 70\% in Auts2-KO and Nrf1-KO, respectively, Figure 6B). Moreover, a slight decrease in the percentage of terminally differentiated MN in Auts2-KO (32\% to 25\%) and a more severe defect in Nrf1-KO (32\% to 16\%) were observed (Figure 6B), indicating that Auts2-KO and Nrf1-KO result in a defect in PNP differentiation into MN. To gain more insight into the molecular mechanism by which AUTS2 and NRF1 contribute to the transition from PNP to MN, we further analyzed the differentially expressed genes (DEGs) specifically in the MN population from either WT or Auts2-KO (see Methods). Among the top 500 DEGs identified from WT MN and Auts2-KO MN, 458 genes were down-regulated in Auts2-KO, in accordance with the role of AUTS2 in transcriptional activation (Figures 6C, 1G and 5B)(Gao et al., 2014). Furthermore, about half of these 458 genes (205 of 458) were also down-regulated in Nrf1-KO MN compared to WT MN (C1, C2 and C3 labeled on the right, Figure $6 \mathrm{C}$ ). To test whether NRF1-directed AUTS2 binding is required for the transcriptional activation of AUTS2-ncPRC1-associated active genes (cluster3 region, Figures $5 \mathrm{C}$ and 5G), we compared the differentially expressed genes in Nrf1-KO MN with the genes located in cluster3 and cluster1 regions from Ring1B ChIP-Seq. Consistent with the loss of AUTS2- 
ncPRC1 binding to active genes upon NRF1 knockout (Figures $5 C$ and $5 G$ ), genes located in cluster3 regions were enriched in the down-regulated category instead of being up-regulated in Nrf1-KO MN (Figure S7). In contrast, very few of the genes located in cluster1 region (corepressed by CPRC1 and PRC2) are affected in Nrf1-KO MN (Figure S7). These results strongly suggest that AUTS2 and NRF1 coordinately function in regulating transcriptional activation (Figures 5C and 5G).

We next asked whether the down-regulated genes in Auts2-KO MN reflected the WT transition from PNP to MN. Indeed, 186 of the 458 genes were normally up-regulated during PNP to MN differentiation in WT, but were defective in activation in Auts2-KO (C1, C2 and C3 labeled on the left, Figure 6C). For example, up-regulation of Asic2, a member of the sodium channel superfamily that regulates synaptic function(Zha et al., 2009) and of Pnpla6, a phospholipase that functions in neurite outgrowth(Guerreiro et al., 2015) were significantly attenuated under conditions of AUTS2 depletion. Finally, genes down-regulated in Auts2-KO MN were enriched for GO terms related to neuronal differentiation and function (Figure 6D). These data demonstrate that AUTS2 and NRF1 function coordinately to foster the appropriate differentiation of posterior neural progenitors to motor neurons by directly binding to and activating a subset of the relevant genes (Figure 6E). 


\section{DISCUSSION}

The results herein point to an instrumental role for the transcription factor NRF1 in facilitating chromatin access to the ncPRC1.3 comprising AUTS2, P300, CK2, PCGF3, and RYBP or YAF2. The function of this particular ncPRC1 has been converted from that of the typical PRC1 in facilitating transcription repression to that of a transcriptional activator(Gao et al., 2014). This conversion involves AUTS2-mediated recruitment of the P300 transcriptional co-activator and CK2-mediated phosphorylation of serine 168 of the integral PRC1 subunit, RING1, thereby thwarting PRC1-mediated monoubiquitination of H2AK119(Gao et al., 2014). Given that RYBP/YAF2 within other ncPRC1 complexes stimulate such RING1A/RING1B-mediated ubiquitination, their presence within ncPRC1.3 may indicate additional RYBP/YAF2 function(s). Importantly, as shown here, most of the genomic sites occupied by AUTS2 require NRF1, while most NRF1 sites are AUTS2-independent.

We noticed that the motif of transcription factor NRF1 is also significantly enriched in AUTS2bound regions in a previous report(Oksenberg et al., 2014), although the enrichment is less dramatic than observed here. A recent study identified transcription factor USF1/2 as being key to PCGF3 chromatin binding in $\mathrm{mESC}$ (Scelfo et al., 2019), yet the motif corresponding to the DNA binding site of USF1/2 was not recovered in our study, suggesting that cell type/tissue specific mechanisms might dictate ncPRC1.3 recruitment to chromatin.

Instead, our findings direct attention to NRF1 in facilitating chromatin access by AUTS2, which is pivotal to the role of AUTS2 at a subset of genes involved in neurodevelopment. NRF1 has previously been shown to exhibit dimerization and to be subject to phosphorylation at several serine residues in its amino-terminus(Gugneja and Scarpulla, 1997). These phosphorylation events do not regulate NRF1 dimerization, but instead mutation of these sites compromise NRF1 DNA binding activity(Gugneja and Scarpulla, 1997). This report also indicates that CK2 could stimulate the DNA binding activity of NRF1 in vitro(Gugneja and Scarpulla, 1997). As CK2 is an integral component of ncPRC1.3 and inhibits its repressive activity by phosphorylating its RING1A/B component(Gao et al., 2014), the presence of CK2 might also foster NRF1 activity to promote ncPRC1.3-mediated transcription activation through AUTS2 interaction with P300. We speculate that CK2 exerts such a coordinated function, resulting in the optimal activation of ncPRC1.3-AUTS2 target genes in the brain.

Evidence involving NRF1 have highlighted its importance in mitochondrial biogenesis(Scarpulla, 2011), as well as in retinal development(Hsiao et al., 2013; Kiyama et al., 2018). Intriguingly, the pathways fostering mitochondrial integrity might be critical to those regulating distinct developmental pathways. While little is known about its role in development of the CNS, NRF1 is widely expressed within the CNS as evidenced by the $N r f 1^{\text {LacZ }}$ mouse line (Figure S8B). We attempted to delete NRF1 in the mouse brain from the embryonic stage in order to study NRF1mediated AUTS2 recruitment, but such embryos did not survive, consistent with a previous report that Nrf1-null mouse embryos die between embryonic day 3.5 (E3.5) and E6.5(Huo and Scarpulla, 2001). Instead, we chose the Tbr1 ${ }^{\text {CreERT2 }}$ line to strategically delete NRF1 in the adult 
mouse brain to examine the role of NRF1 in the CNS (Figures S8A-C). Importantly, we noticed several histological anomalies in these mutant mice $\left(T b r 1^{\mathrm{CreERT2/+}}\right.$ : $\mathrm{Nrf1}{ }^{\mathrm{fx} / \mathrm{fx}}$ : Pou $4 f 1^{\mathrm{CKO} /+}$ ), compared to control mice $\left(\mathrm{Tbr} 1^{\mathrm{CreERT2/+}}: \mathrm{Nrf} 1^{\mathrm{fx} /+}:\right.$ POu $\left.4 f 1^{\mathrm{CKO} /+}\right)$, including a reduction in the size of the hippocampus and the width of the corpus callosum, as well as an enlarged lateral ventricle, indicating neuronal loss in both the cortex and hippocampus (Figures S8D and S8E). As well, a significant loss in retinal ganglion cells (RGCs) is observed in retinas collected from these mutant mice (Figures S8F and S8G).

It is important to emphasize that the reduced volume of corpus callosum observed in NRF1 mutant $\left(T b r 1^{\text {CreERT2/+ }}: \mathrm{Nrf1}^{f \times / f x}\right.$ : Pou $\left.4 f 1^{\mathrm{CKO} /+}\right)$ mice is a key syndromic feature observed in RSTS patients(Cantani and Gagliesi, 1998), as well as in patients harboring mutations in the AUTS2 HX repeat domain as reported here, strongly supporting that NRF1 and AUTS2 function coordinately in regulating brain development. The corpus callosum (CC) connects the cerebral hemispheres and is the largest fiber tract in the brain(Edwards et al., 2014). During development, defects in neurogenesis, telencephalic midline patterning, neuronal migration and specification, axon guidance and post-guidance development can interrupt CC formation(Reyes et al., 2020).

Our findings also point to an instrumental role for the AUTS2 HX repeat domain given its requirement for AUTS2 interaction with P300/CBP. Importantly and as shown here, AUTS2 variants in this domain exhibit profound clinical and transcriptional effects in vivo. Given the existence of ncPRC1.3 in which AUTS2 conveys transcription activation, along with the reported association of AUTS2 haploinsufficiency in AUTS2-syndrome(Beunders et al., 2013), and possibly in Autism Spectrum Disorders(Sultana et al., 2002), AUTS2 had appeared key for modulating appropriate neurodevelopment. Here, we identified a critical role for its HX repeat domain in mediating AUTS2 interaction with P300 and resultant transcriptional activation by interrogating mutations found in individuals exhibiting a distinct and severe neurodevelopmental syndrome that overlaps with RSTS. Our in vitro and cell-based studies demonstrate that RSTS-associated AUTS2 mutations in the HX repeat domain, but not a mutation outside this region, disrupt AUTS2-P300 interaction and attenuate AUTS2-mediated active transcription. Of note, a recent study reports a patient with a syndromic neurodevelopmental disorder harboring a different mutation (532-541 aa deletion) in the AUTS2 HX repeat domain(Martinez-Delgado et al., 2020), further pointing to the critical role of AUTS2 in normal brain functioning. Moreover, mutations within exon 9 outside the HX repeat such as the PY motif, as well as a mutation at residue 495, result in individuals that display severe behavioral phenotypes such as epilepsy, in lieu of RSTS (Table S1); further stressing the role of AUTS2 in normal brain function. Consistent with the notion that ncPRC1.3 is important in the brain, mutations in FBRSL1(Ufartes et al., 2020), which binds competitively with AUTS2 to ncPRC1.3, are also associated with a neurodevelopmental syndrome. However, it is not yet known whether FBRSL1-ncPRC1.3 acts in a repressive or activating manner.

Taken together, our findings support a model in which NRF1 is a key factor that directs AUTS2nCPRC1.3 binding to a subset of neuronal differentiation-related genes that are thereby subjected to activation by AUTS2 interaction with P300 through the AUTS2 HX repeat domain 
(Figure S9). As shown here, ablation of NRF1 or AUTS2 leads to defective progenitor to motor neuron differentiation in vitro. Deletion of NRF1 in the mouse brain and mutations in the AUTS2 $\mathrm{HX}$ repeat domain in humans show CC malformation, a common feature of RSTS patients which is largely associated with specific pathogenic variants in the EP300/CREBBP genes (Figure S9). The precise mechanisms by which NRF1 and AUTS2-ncPRC1.3 coordinately regulate gene expression, neuronal differentiation and thus mouse and human brain development and function in post-natal individuals require further investigation. Alternate approaches need to be explored as deletion of either NRF1 or AUTS2 (both long and short isoforms) apparently lead to early embryonic lethality. The generation of mouse models carrying mutations in the AUTS2 HX repeat domain might expedite future studies should they recapitulate the RSTS phenotype or other neurological diseases. 
A

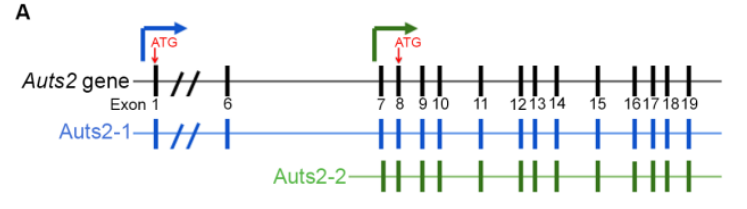

。

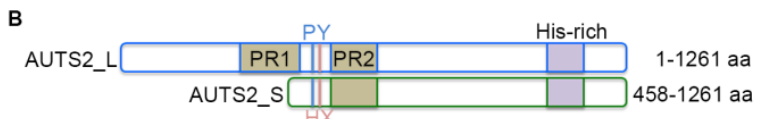

C D
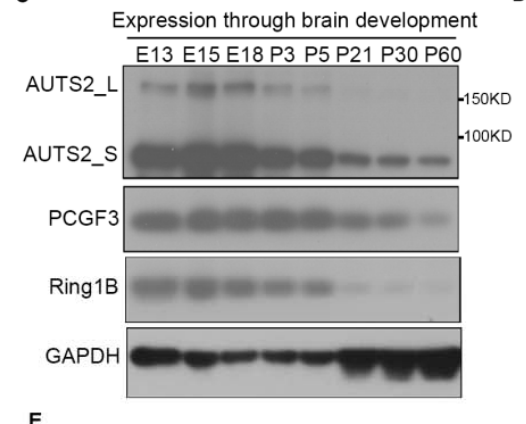

E

\begin{tabular}{|c|c|}
\hline & IP-MS: AUTS2 \\
\hline AUTS2 & 59 \\
\hline RNF2 & 20 \\
\hline Csnk2a1 & 20 \\
\hline DCAF7 & 18 \\
\hline PCGF3 & 16 \\
\hline Ring1 & 13 \\
\hline Csnk2a2 & 7 \\
\hline Csnk2b & 6 \\
\hline RYBP & 2 \\
\hline PCGF5 & 1 \\
\hline
\end{tabular}

G
F
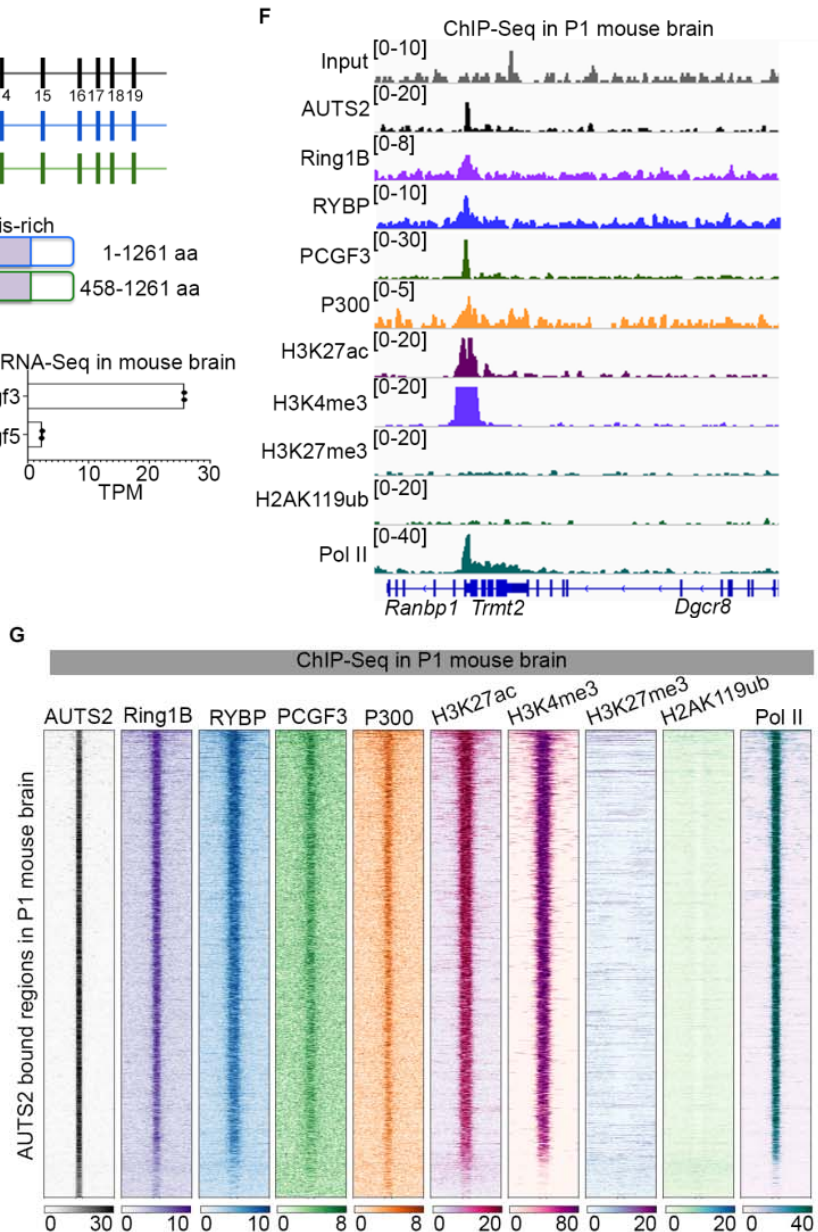

Figure 1. AUTS2-ncPRC1.3 targets active genes in mouse brain

(A) Schematic showing the mouse Auts2 gene structure and its two major transcripts in the mouse brain. Red arrows indicate the translational start codon used for each transcript.

(B) Schematic showing the domains of the long and short isoforms of mouse AUTS2 protein. PR, proline-rich region; PY, PPPY motif; $\mathrm{HX}, \mathrm{HX}$ repeat motif, comprising alternating $\mathrm{HQ}(x 6)$ or HT $(\times 3)$ residues; His-rich, eight histidine repeats.

(C) Expression of AUTS2 and core nCPRC1.3 components in the mouse brain. Immunoblotting was performed with whole brain extracts at various developmental stages, as indicated.

(D) Bar graphs showing the value of transcripts per kilobase million (TPM) for Pcgf3 and Pcgf5 revealed by RNA-Seq performed from whole brain lysate at postnatal day 1.

(E) Proteomic mass spectrometry results of immunoprecipitation (IP) using AUTS2 antibody in whole brain lysate at postnatal day 1.

(F) IGV browser views showing ChIP-seq profile for input, AUTS2, RING1B, RYBP, PCGF3, P300, H3K27ac, H3K4me3, H3K27me3, H2AK119ub1 and RNA Polymerase II (PollI) at the representative loci. ChIP-seq was performed in whole brain lysate at postnatal day 1.

(G) Heatmap showing AUTS2, RING1B, RYBP, PCGF3, P300, H3K27ac, H3K4me3, H3K27me3, H2AK119ub1 and RNA Polymerase II ChIP-seq signals centered on AUTS2 bound regions ( $\pm 5 \mathrm{~kb}$ ). ChIP-seq was performed in whole brain lysate at postnatal day 1. 

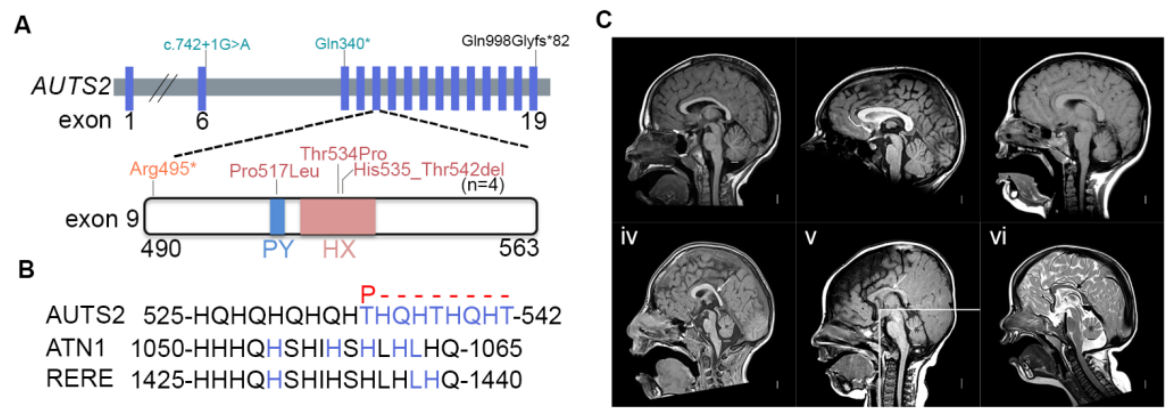

Figure 2. Patients with mutations in AUTS2 HX repeat have features that overlap with Rubinstein-Taybi syndrome.

(A) Schematic illustrating mutations in the AUTS2 gene from individual patients as identified through trio-based exome sequencing. Mutations resulting in similar clinical features are labeled with the same color. PY, PPPY motif; HX, HX repeat motif, comprising alternating $\mathrm{HQ}$ (x6) or $\mathrm{HT}(\mathrm{x} 3)$ residues.

(B) Mutations in the HX repeat of AUTS2 compared with those reported for ATN1 and RERE. The mutated residues are labeled in blue. The variant substitutions for AUTS2 present in affected individuals are indicated above the sequence alignment (red).

(C) Magnetic resonance images from 5 individuals with missense variants in AUTS2 exon 9 and a normal control. The three midline sagittal images in the top row all show normal midline structures. They come from a normal control (i) a girl with a missense variant ( $p$.Thr534Pro) in the PY motif (ii) and a boy with the recurrent INDEL (p.His535_Thr542 del) in the HX motif (iii). The three midline sagittal iimages in the bottom row come from a boy with a missense mutation in the HX domain (p.Thr534Pro) and two boys with the recurrent INDEL in the HX motif. All three show thin show thin and dysplastic corpus callosa (arrows in iv-vi), and small cerebellar vermis (asterisks in iv-vi). The horizontal white or black lines mark the level of the obex, the usual lower extend of the vermis. 
A

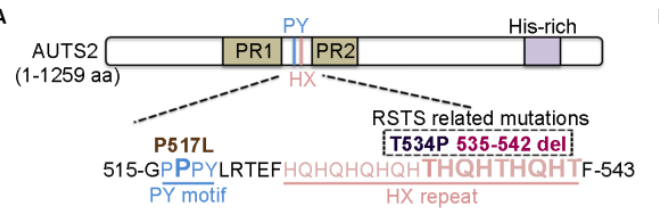

B

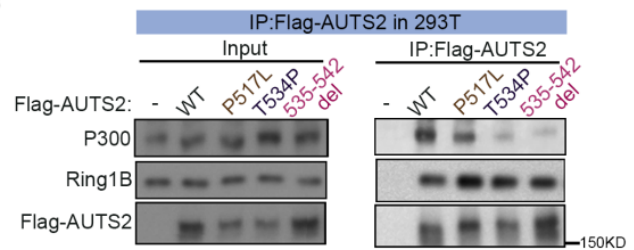

C

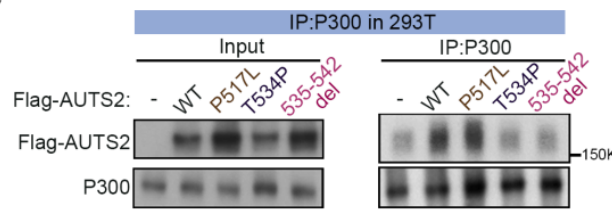

$\mathrm{F}$

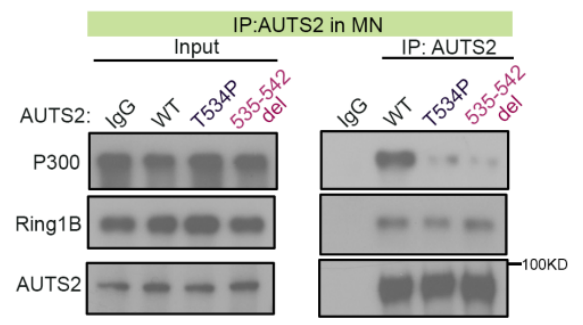

D

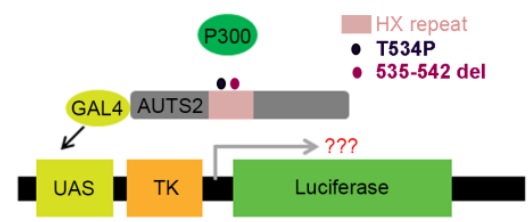

E
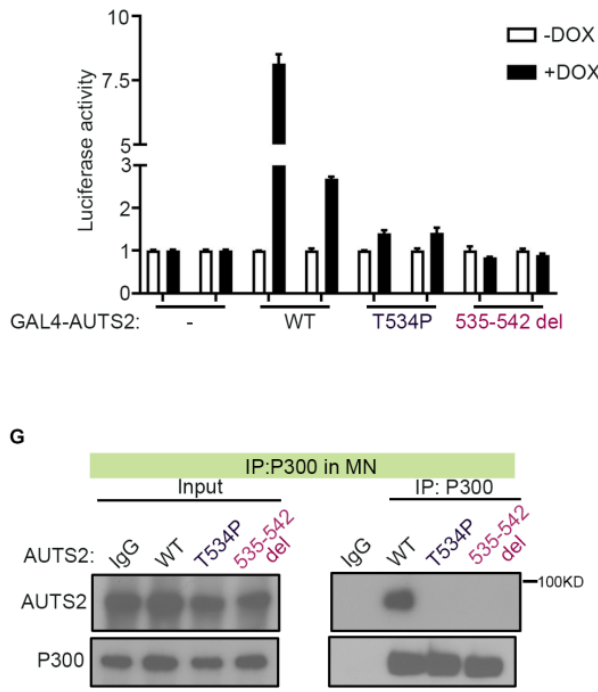

Figure 3. Mutations in AUTS2 HX repeats disrupt both P300 binding and AUTS2-mediated transcription activation.

(A) Schematic showing human AUTS2 variants constructed and expressed in 293 T-REx cells. The two variants within the HX repeat are associated with Rubinstein-Taybi syndrome.

(B-C) Western blots show co-IP results from nuclear extract of 293 T-REx cells expressing FlagAUTS2, either WT or mutant versions as indicated, using Flag antibody (B) or reciprocal IP using P300 antibody (C).

(D) Schematic of the reporter construct for the luciferase assay in the context of GAL4-AUTS2, either WT or mutant versions as indicated.

(E) Luciferase activity in cells expressing GAL4-AUTS2, either WT or mutant versions before and after doxycycline treatment.

(F-G) Western blots show co-IP results from nuclear extract of motor neuron differentiated from WT and Auts2 HX mutant (T534P and 535-542 aa deletion respectively) mESC as indicated, using AUTS2 antibody (F) or reciprocal IP using P300 antibody (G). 
A Motif analysis
C्CCCATCCCAT ${ }^{2}$ motif in AUTS2 peaks
GCCATCCCCAC motif for NRF1

B

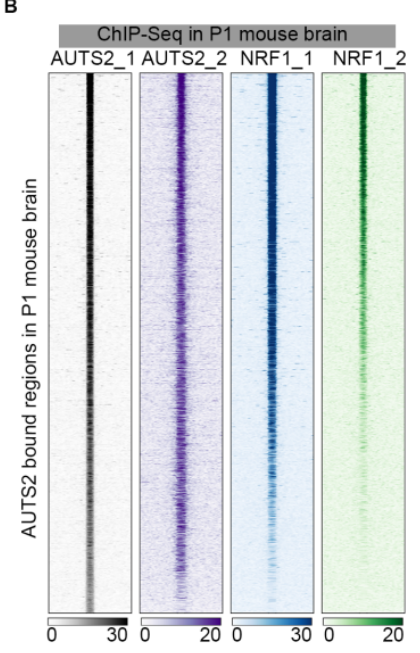

c

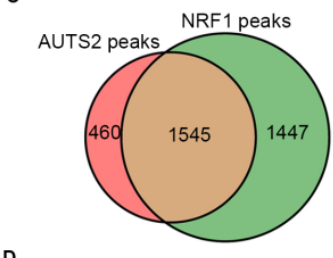

D

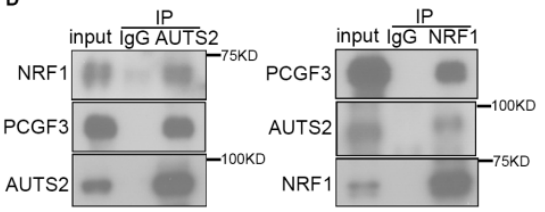

E

Expression throuh brain development

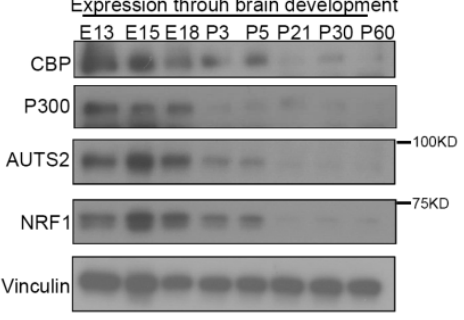

Figure 4. Transcription factor NRF1 colocalizes with AUTS2 on chromatin and physically interacts with AUTS2 in mouse brain

(A) Top, motif analysis of AUTS2-bound regions in mouse brain using HOMER. Bottom, known motif for transcription factor NRF1.

(B) Heatmap showing AUTS2 and NRF1 ChIP-seq signals centered on AUTS2-bound regions $( \pm 5$

kb) with two replicates. ChIP-seq was performed in whole brain lysate at postnatal day 1 .

(C) Venn diagram showing the extent of overlap for AUTS2- and NRF1-bound regions revealed by ChIP-seq performed in whole brain lysate at postnatal day 1.

(D) Reciprocal co-immunoprecipitation and western blot analyses of AUTS2 and NRF1 interaction in whole brain lysate at postnatal day 1.

(E) Expression of CBP, P300, AUTS2 and NRF1 in mouse brain. Immunoblotting was performed with whole brain extracts at various developmental stages, as indicated. 
A

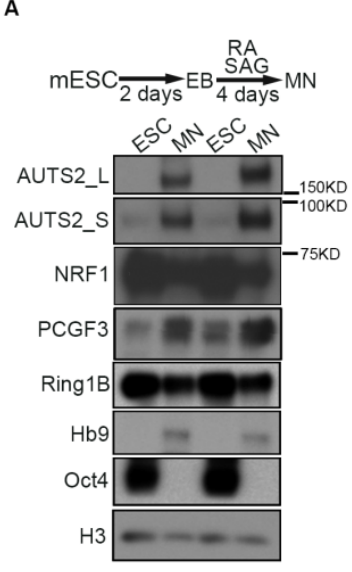

B

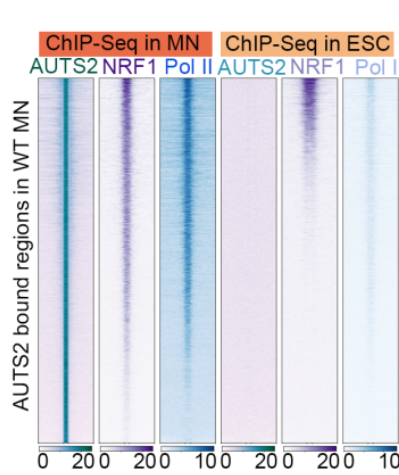

D

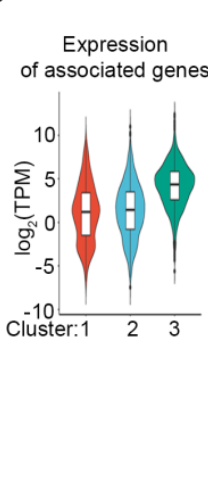

$\mathrm{E}$

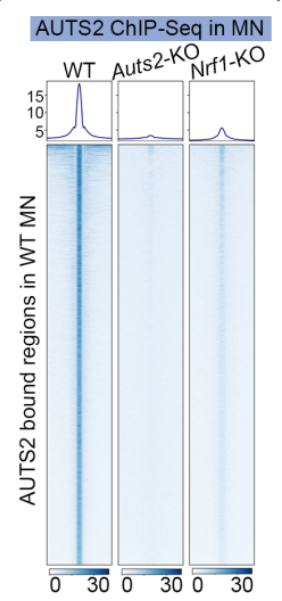

$\mathbf{F}$

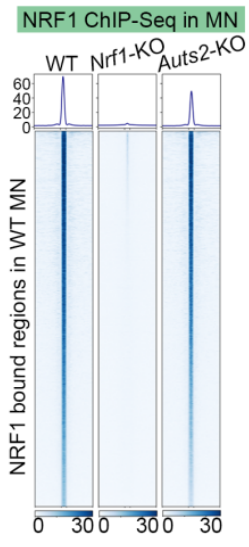

c
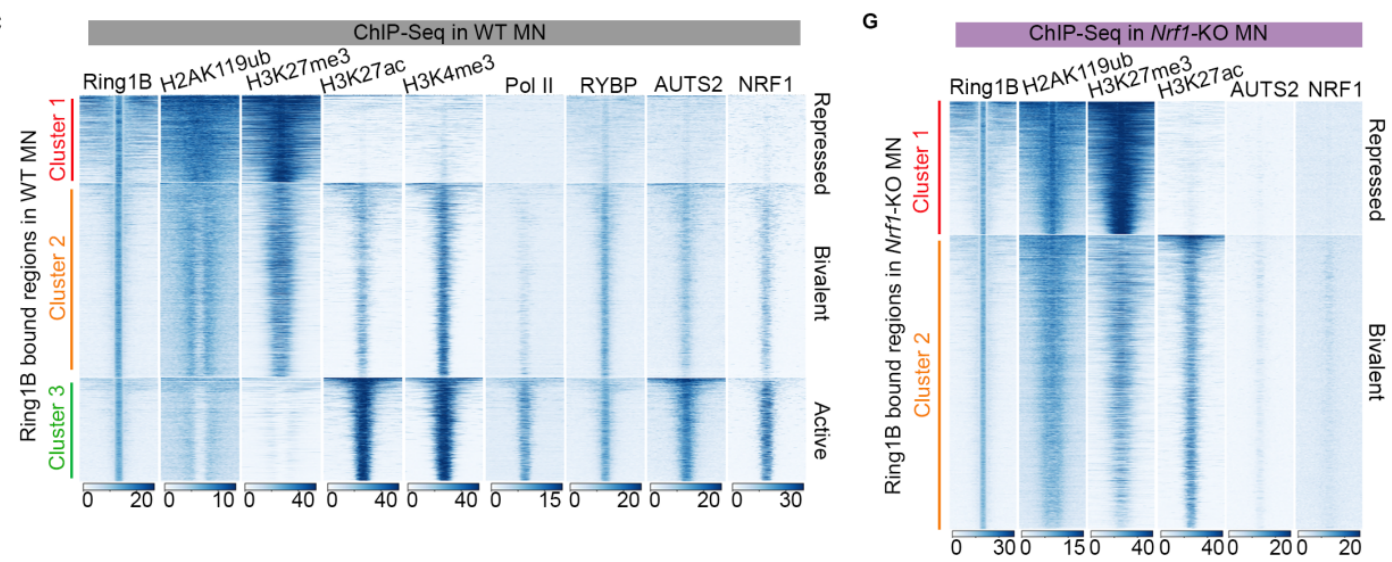

Figure 5. NRF1 is crucial for AUTS2-ncPRC1.3 associated active transcription in MN

(A) The schematic at top depicts the protocol for differentiation of mouse embryonic stem cells (mESC) to motor neurons (MN) using retinoic acid (RA) and smoothened agonist (SAG). EB, embryoid bodies. Below is a western blot showing the expression of AUTS2, NRF1, PCGF3 and Ring1B in ESC and MN.

(B) Heatmap showing AUTS2, NRF1 and RNA Pol II ChIP-seq signals centered on AUTS2 bound regions identified in WT MN ( $\pm 5 \mathrm{~kb})$. ChIP-seq was performed in ESC and MN.

(C) k-means clustering of RING1B, H2AK119ub, H3K27me3,H3K27ac, H3K4me3, RNA Pol II, RYBP, AUTS2 and NRF1 ChIP-seq signals from WT MN centered on Ring-B bound regions identified in WT MN $( \pm 5 \mathrm{~kb})$.

(D) Violin plot of the $\log _{2}$ (TPM) of the genes assigned to each cluster [as indicated in (C)], quantified from RNA-seq in WT MN.

(E) Average density profiles (top) and heatmap (bottom) showing AUTS2 ChIP-seq signals from WT, Auts2-KO and Nrf1-KO MN centered on AUTS2-bound regions identified in WT MN ( $\pm 5 \mathrm{~kb})$. (F) Average density profiles (top) and heatmap (bottom) showing NRF1 ChIP-seq signals from WT, Nrf1-KO and Auts2-KO MN centered on NRF1-bound regions identified in WT MN ( $\pm 5 \mathrm{~kb}$ ). (G) k-means clustering of RING1B, H2AK119ub, H3K27me3, H3K27ac, AUTS2 and NRF1 ChIP-seq signals from Nrf1-KO MN centered on Ring1B-bound regions identified in Nrf1-KO MN ( $\pm 5 \mathrm{~kb})$. 


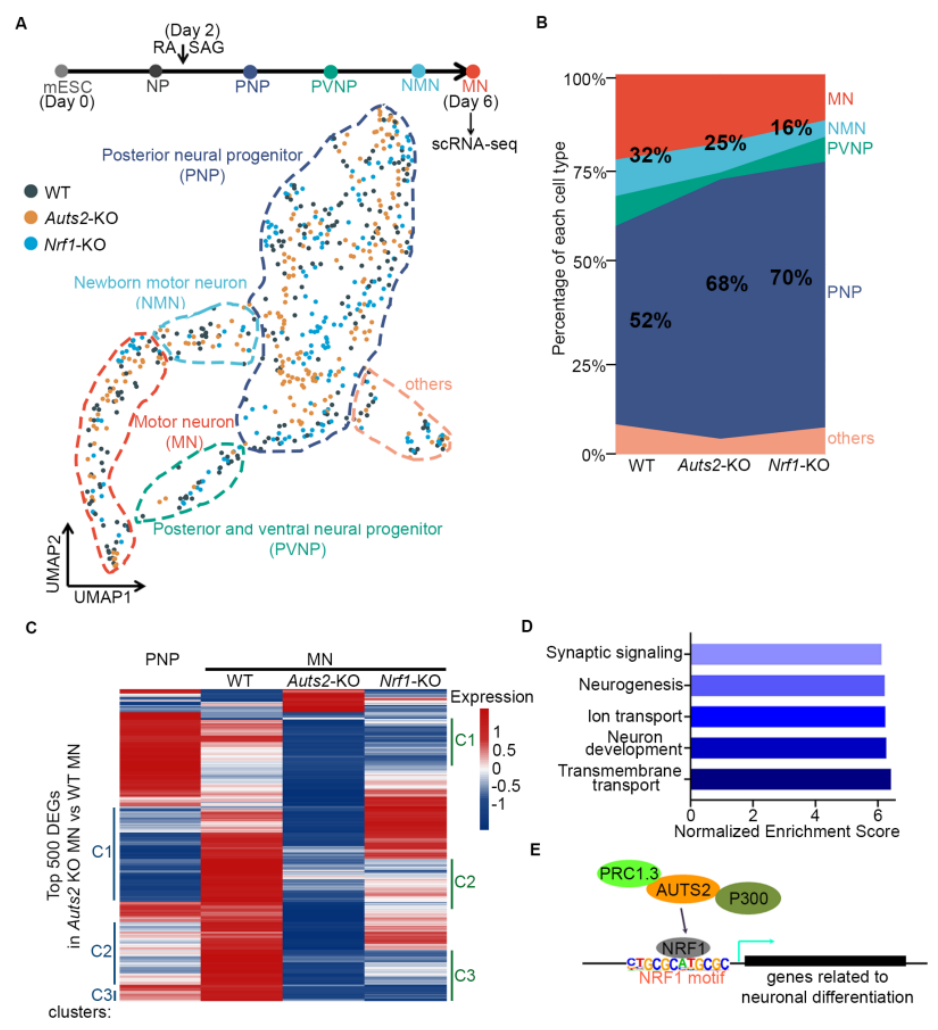

Figure 6. Defect in posterior neural progenitor differentiation into motor neuron in the absence of AUTS2 or NRF1 by SCRNA-Seq

(A) The schematic at top depicts cell lineage transitions from mouse embryonic stem cells (mESC) to motor neurons (MN). MN (day 6) differentiated from WT, Auts2 knockout and Nrf1 knockout ESC were harvested for sCRNA-Seq. Below is the dimensionality reduction (UMAP) of 632 cells from WT, Auts2-KO and Nrf1-KO samples, sequenced with the Smart-seq3 technique and colored by sample identity (WT, 228 cells; Auts2-KO, 221 cells; Nrf1-KO, 183 cells). Five cell classes revealed by unsupervised clustering of cellular transcriptomics are represented by colorcoded circles of dashed-lines and annotated based on marker gene expression. Colors of dashed-lines match those for the cell types shown at top.

(B) Proportional stacked area graph showing the abundances of each cell type in $\mathrm{MN}$ differentiated from WT, Auts2-KO and Nrf1-KO ESC. The percentage of motor neuron $(\mathrm{MN}+\mathrm{NMN})$ and posterior neural progenitor (PNP) in each sample are labelled.

(C) Top 500 differentially expressed genes (DEGs) were identified by comparing Auts2-KO MN versus WT MN. Expression of these DEGs across WT PNP, WT MN, Auts2-KO MN and Nrf1-KO $\mathrm{MN}$ is shown by heatmap. Color scale represents the averaged and scaled expression values from each cell population. Labelling of clusters $(C 1, C 2, C 3)$ on left is based on gene expression differences in WT PNP, WT MN and Auts2-KO MN. Labelling of clusters (C1, C2, C3) on right is based on gene expression differences in WT MN, Auts2-KO MN and Nrf1-KO MN.

(D) Bar plot summarizing results of gene set enrichment analysis for genes downregulated in Auts2 KO MN versus WT MN.

(E) Model depicting transcription factor NRF1-mediated recruitment of AUTS2-ncPRC1.3 resulting in activation of genes related to neuronal differentiation. 
bioRxiv preprint doi: https://doi.org/10.1101/2021.03.30.437620; this version posted May 4, 2021. The copyright holder for this preprint (which was not certified by peer review) is the author/funder, who has granted bioRxiv a license to display the preprint in perpetuity. It is made available under aCC-BY-NC-ND 4.0 International license.

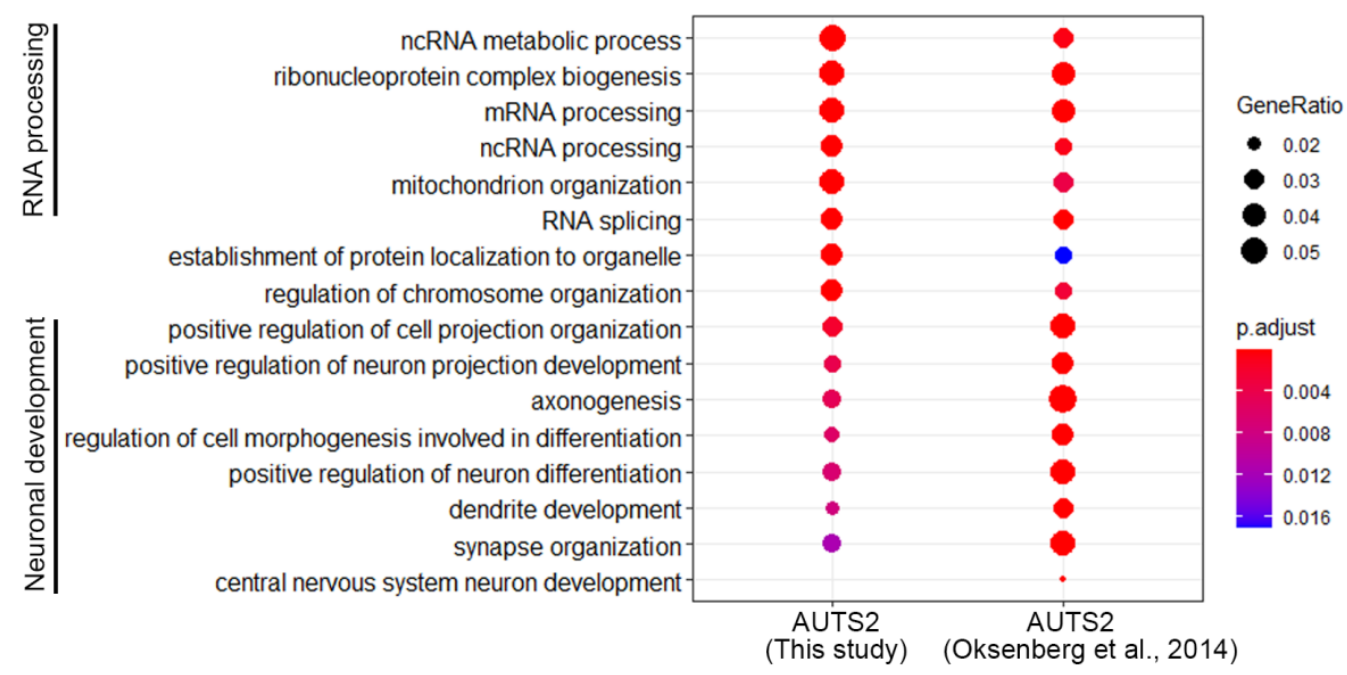

Figure S1. GO analysis of AUTS2-bound regions in mouse brain from our study and a study from(Oksenberg et al., 2014), related to Figure 1. 
bioRxiv preprint doi: https://doi.org/10.1101/2021.03.30.437620; this version posted May 4, 2021. The copyright holder for this preprint (which was not certified by peer review) is the author/funder, who has granted bioRxiv a license to display the preprint in perpetuity. It is made available under aCC-BY-NC-ND 4.0 International license.

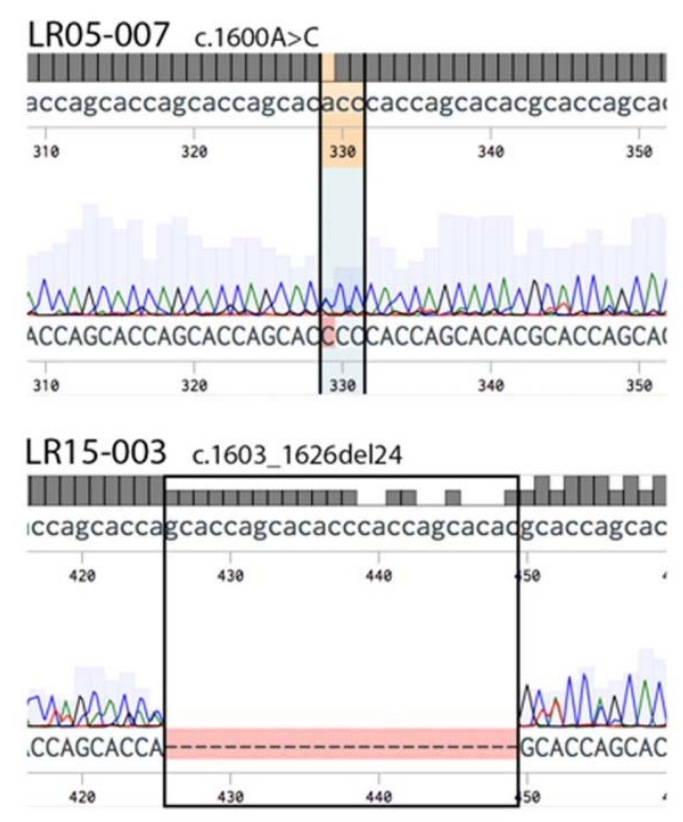

Figure S2. Chromatograms showing Sanger sequencing confirmation of AUTS2 mutations in cDNA amplified from patient fibroblasts that were initially detected by exome sequencing of genomic DNA, related to Figure 2. 
A

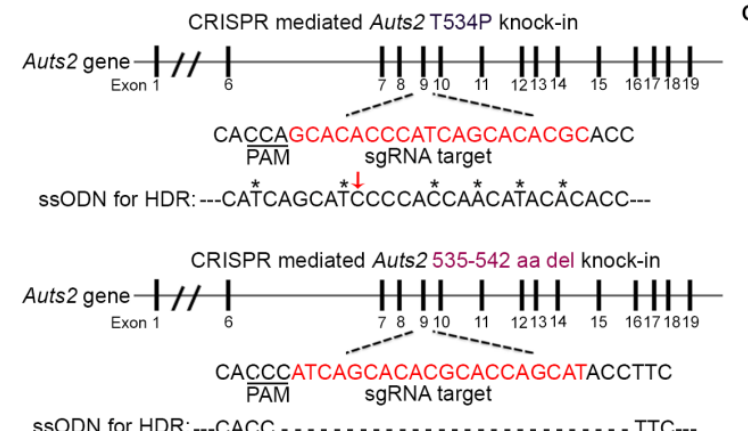

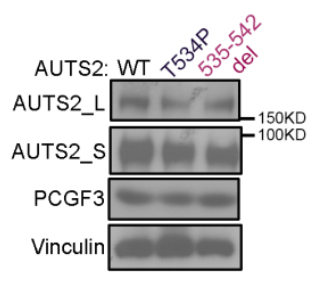

Figure S3. CRISPR-Cas9-mediated knock-in of mutations in AUTS2 HX repeat domain in mESC, related to Figure 3.

(A) The design of sgRNA and single stranded oligodeoxynucleotide (ssODN) donor used for Auts2 T534P knock-in with CRISPR-Cas9. Red arrow indicates T534P mutation and asterisk*denotes the nucleotide substitution to avoid CRISPR targeting.

(B) The design of sgRNA and single stranded oligodeoxynucleotide (sSODN) donor used for Auts2 535-542 aa deletion with CRISPR-Cas9. The 24 bps for coding AUTS2 535-542 aa were deleted in SSODN.

(C) Western blot showing the expression of AUTS2 and PCGF3 in motor neuron differentiated from WT and Auts2 HX mutant (T534P and 535-542 aa deletion respectively) mESC as indicated. 
A
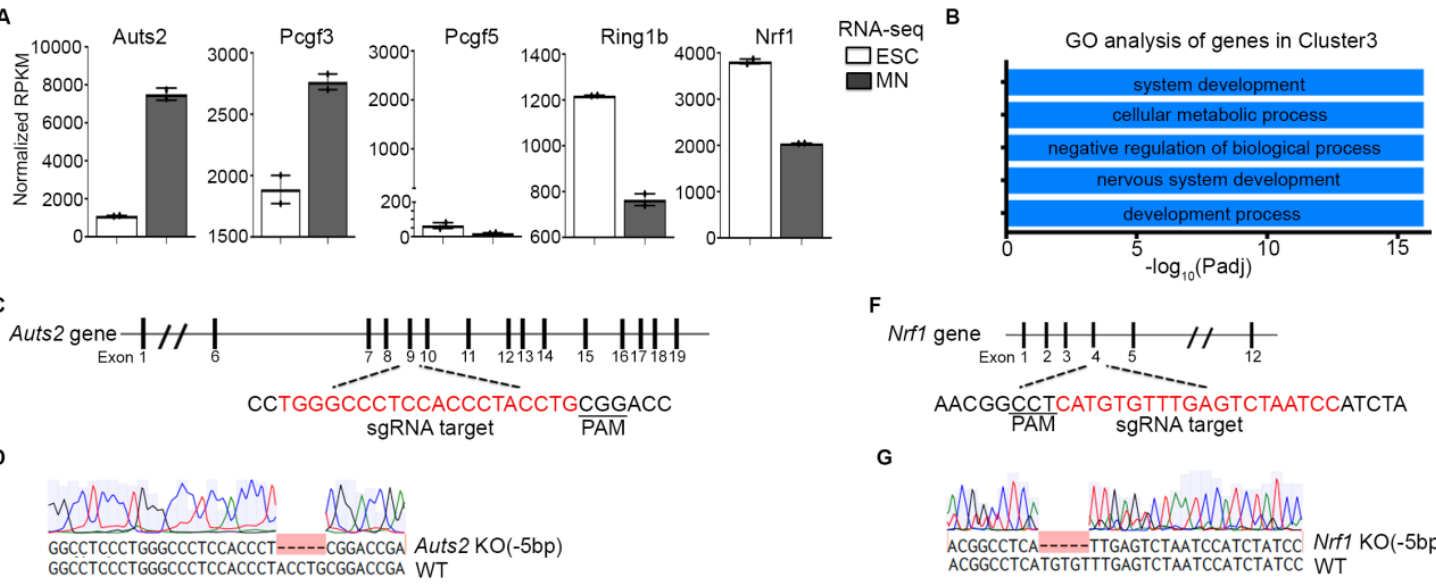

E
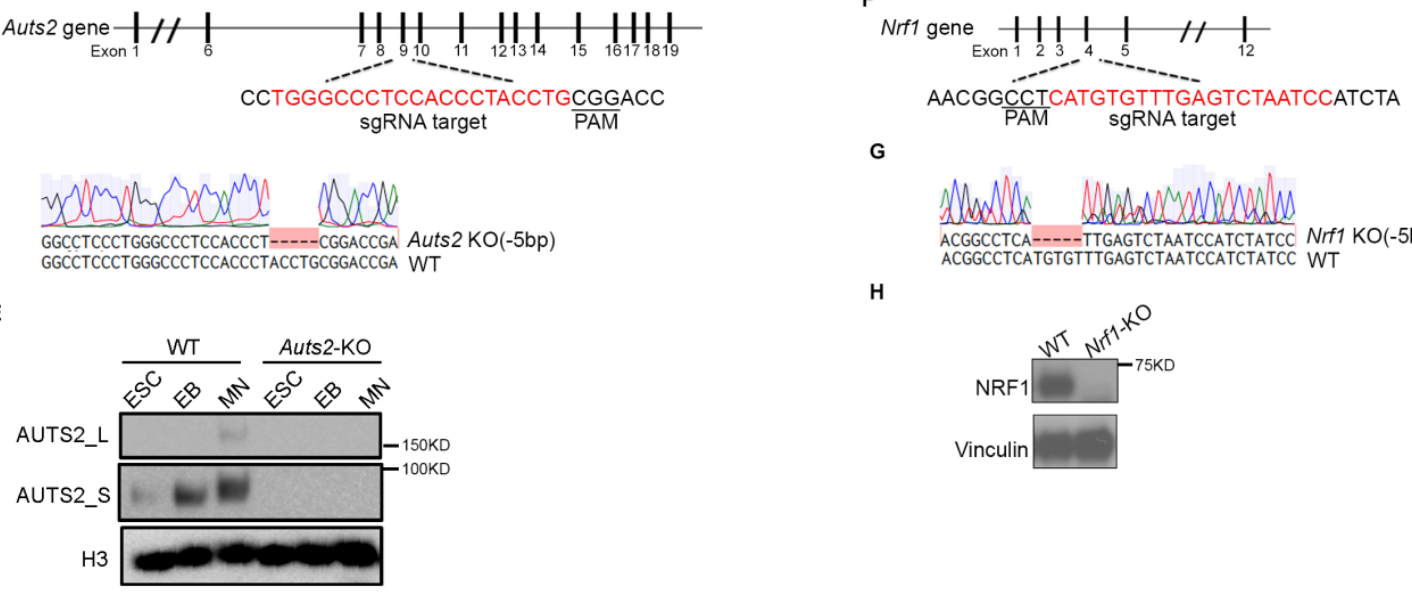

G

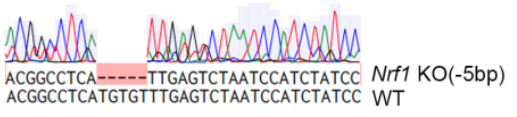

H

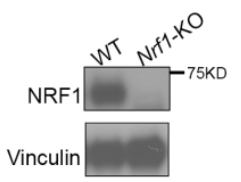

Figure S4. Design and validation of Auts2 and Nrf1 knockout, related to Figure 5.

(A) Bar graphs showing the value of RPKM for Auts2, Pcgf3, Pcgf5, Ring1b and Nrf1 revealed by RNA-Seq performed on ESC and MN.

(B) GO analysis of genes associated with cluster 3 regions (as indicated in Figure $3 \mathrm{C}$ ) from Ring1B ChIP-Seq in MN.

(C-E) Design (C), sanger sequencing (D) and western blot analysis (E) for CRISPR-Cas9 mediated Auts2 knockout.

$(\mathrm{F}-\mathrm{H})$ Design $(\mathrm{F})$, sanger sequencing $(\mathrm{G})$ and western blot analysis $(\mathrm{H})$ for CRISPR-Cas9 mediated Nrf1 knockout. 
A

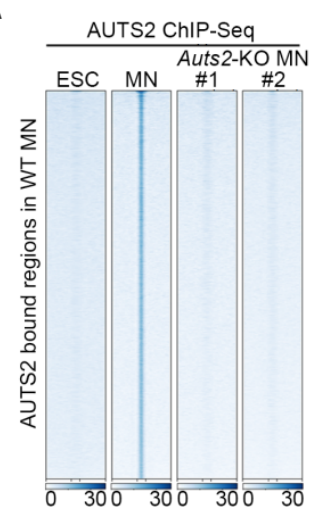

B

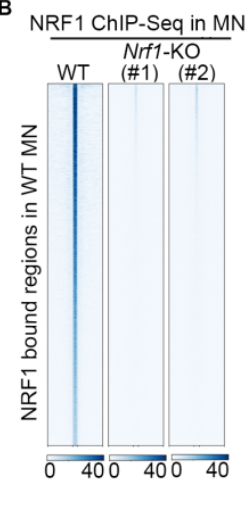

Figure S5. Validation of AUTS2 and NRF1 ChIP-seq specificity under Auts2-KO and Nrf1-KO, related to Figure 5.

(A) Heatmap showing AUTS2 ChIP-seq signals from ESC and either WT or Auts2-KO MN centered on AUTS2-bound regions identified in WT MN ( $\pm 5 \mathrm{~kb}$ ). \#1 and \#2 represent 2 Auts2-KO clones.

(B) Heatmap showing NRF1 ChIP-seq signals from WT and Nrf1-KO ESC centered on NRF1bound regions identified in WT ESC $( \pm 5 \mathrm{~kb})$. \#1 and \#2 represent $2 \mathrm{Nrf1-KO}$ clones. 
A

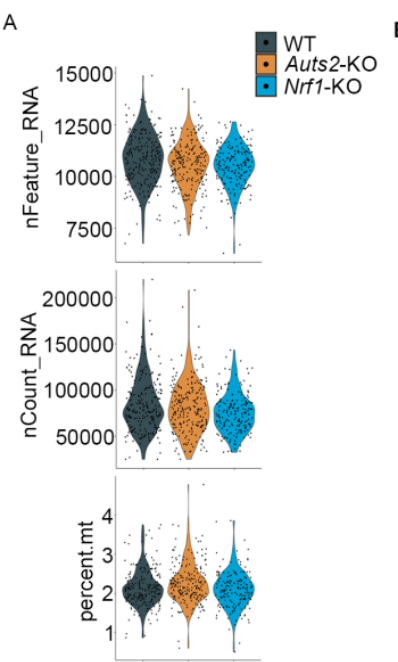

B

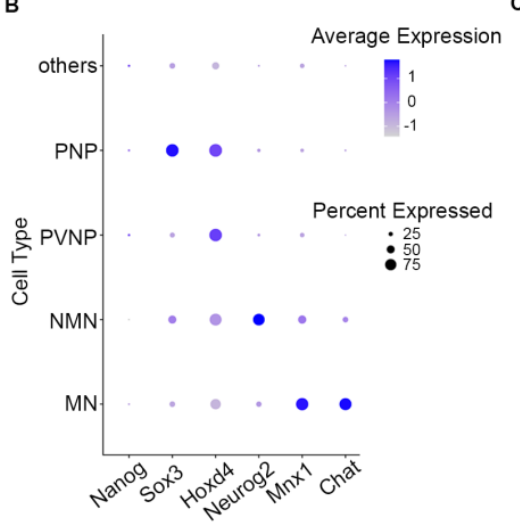

C

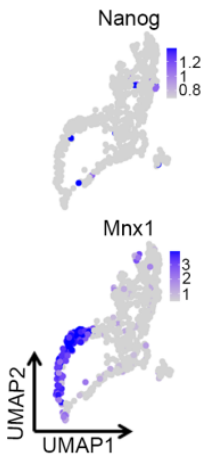

Figure S6. Quality control for scRNA-seq, related to Figure 6.

(A) Violin plots summarizing the number of genes, molecules detected and percentage of reads mapping to mitochondrial genome per cell by scRNA-seq in WT, Auts2-KO and Nrf1-KO samples. (B) Dot plot shows the expression levels and frequencies of cell-type-specific marker genes in each cluster. The size and color of the dot represent the percentage of cells expressing the marker gene and the relative expression level (Z-score) within a cell cluster, respectively.

(C) UMAP plots are colored by the expression level of the genes indicated. Nanog: stem cell marker, Sox3: posterior neural progenitor marker, Neurog2: newborn motor neuron marker, and $M n \times 1$ : motor neuron marker. 


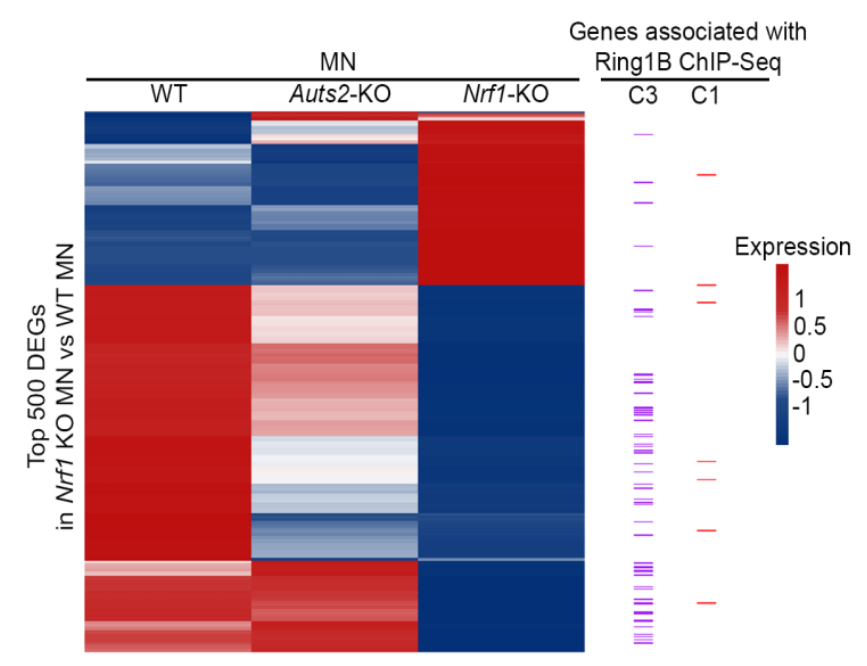

Figure S7. Comparing the differentially expressed genes in Nrf1-KO MN by scRNA-Seq with PRC1 associated active and repressed genes revealed by Ring1B ChIP-Seq, related to Figure 6. Top 500 differentially expressed genes (DEGs) were identified by comparing Nrf1-KO MN versus WT MN. Expression of these DEGs across WT MN, Auts2-KO MN and Nrf1-KO MN is shown by heatmap. Color scale represents the averaged and scaled expression values from each cell population. Each row represents a gene and whether the gene is located in cluster 1 or cluster 3 regions from Ring1B ChIP-Seq (see Figure 5C for more details) is marked on the right. 


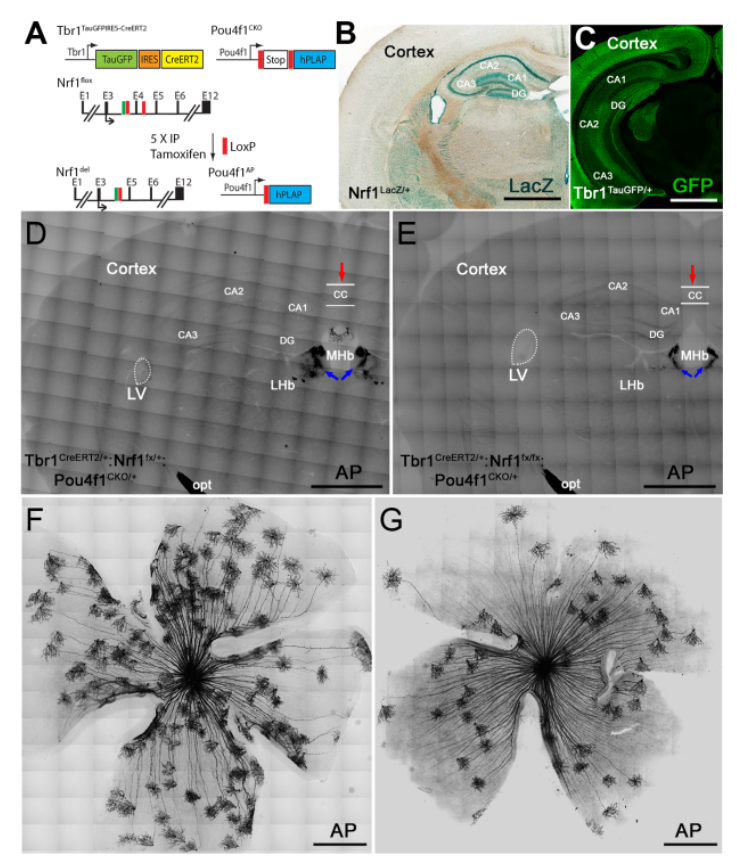

Figure S8. Tbr $1^{\text {CreERT2 }}$-mediated Nrf1-deletion in CNS leading to neuronal loss.

(A) Schematic illustration showing the strategy for Tbr $1^{\text {CreERT2 }}$-mediated conditional KO of exon 4 of the Nrf1 gene. Expression of TauGFP and Cre ${ }^{\text {ERT2 }}$ is driven by the Tbr1 promoter. Double loxP sites were inserted into the third and fourth introns of the Nrf1 gene. Double loxP sites spanning a translation stop codon and followed by the coding region of human placental alkaline phosphatase (AP) were also inserted into the 5' UTR region of the Pou4f1 gene. After Cre ${ }^{\mathrm{ERT} 2}$ activation by tamoxifen, Nrf1 exon 4 will be deleted and hPLAP will be expressed and used for AP staining.

(B) NRF1 expression pattern in adult brain revealed by LacZ expression in the Nrf1 ${ }^{\mathrm{LacZ} /+}$ mouse.

(C) Tbr1 expression pattern as revealed by immunostaining using anti-GFP antibody on Tbr1 $1^{\text {TauGFP/+ }}$ brain section.

(D-E) Representative images of brain sections (at Bregma $-1.7 \mathrm{~mm}$ ) from control (D,

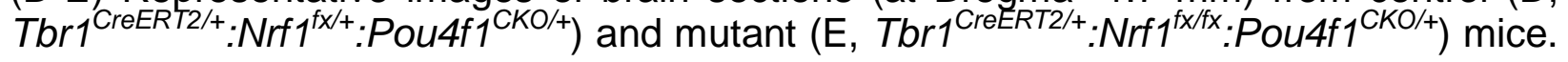
Brain sections were collected three months after intraparietal injection of tamoxifen. A significant reduction in the size of the hippocampus (CA1, CA2, CA3, DG), the width of the corpus callosum (CC, red arrow) and the number of alkaline phosphatase (AP)+ fibers passing through the medium and lateral habenula (MHb, blue arrows), and a significant enlarged lateral ventricle (dotted circle) were observed in the mutant brain section, indicative of neuronal loss upon Nrf1 knockout. CA1-3: hippocampus subregions; DG: dentate gyrus.

(F-G) Representative AP-stained images showing Tbr1-expressing RGC in retinas from

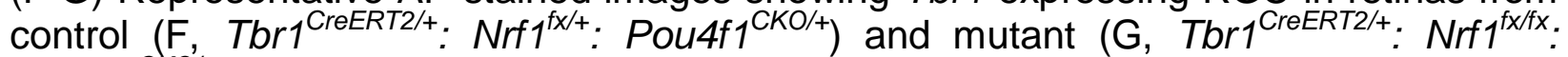
$\mathrm{Pou}_{4 \mathrm{f}} \mathrm{CKO} / \mathrm{H}^{\mathrm{N}}$ mice. Retinas were collected three months after intraparietal injection of tamoxifen. The number of $\mathrm{AP}+\mathrm{RGC}$ in the mutant retinas was reduced by approximately $45 \%$ compared to control $(\mathrm{N}=4$; control: $112.25 \pm 8.30$, mutant: $63.5 \pm$ $11.7 ; \mathrm{P}=0.0005)$, further confirming neuronal loss in the central nervous system upon Nrf1 knockout. Scale bars: $1 \mathrm{~mm}$ in B-E, $500 \mu \mathrm{m}$ in F and G. 
bioRxiv preprint doi: https://doi.org/10.1101/2021.03.30.437620; this version posted May 4, 2021. The copyright holder for this preprint (which was not certified by peer review) is the author/funder, who has granted bioRxiv a license to display the preprint in perpetuity. It is made available under aCC-BY-NC-ND 4.0 International license.

A

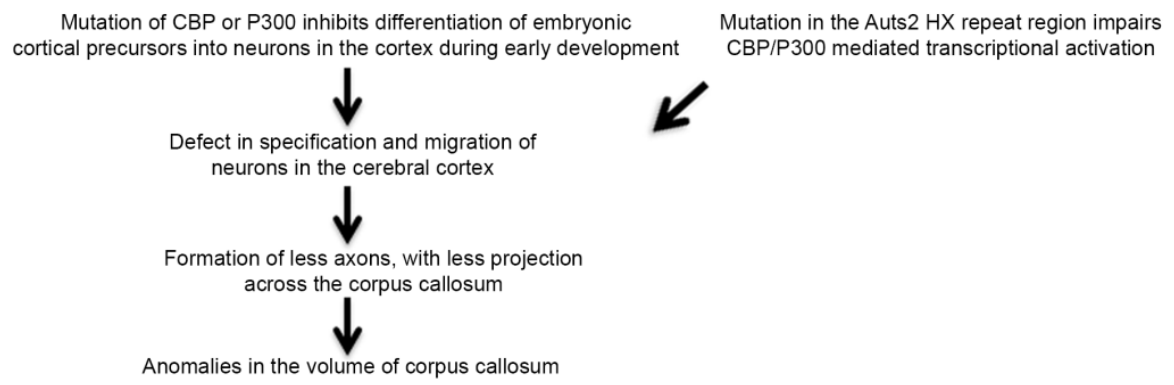

B

Mutations in AUTS2 $\mathrm{HX}$ repeat

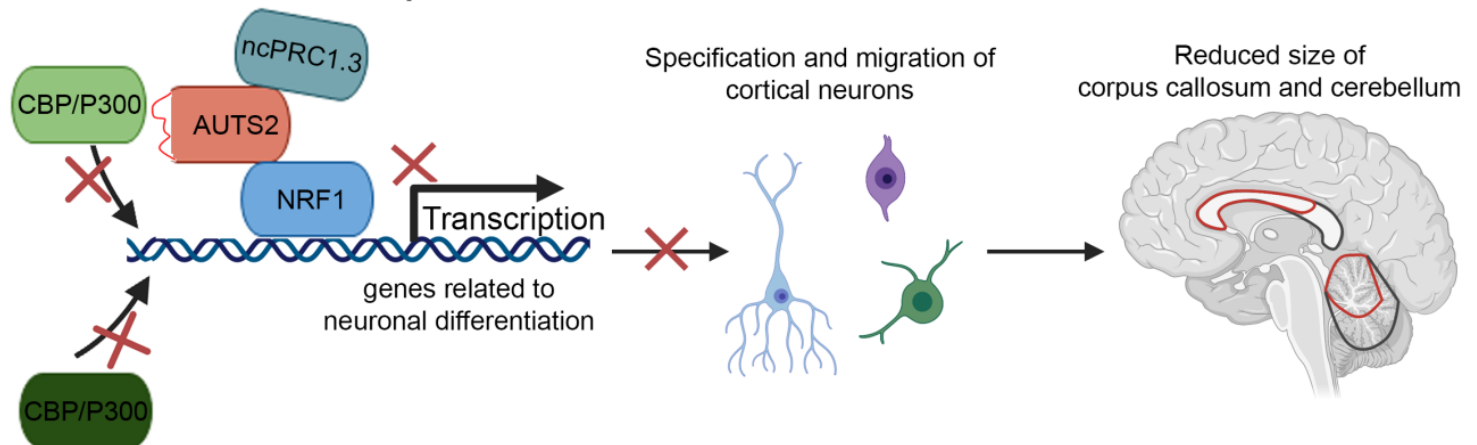

Mutations in CREBBP or EP300 gene

Figure S9. Hypothetical model for associating mutations in AUTS2 HX repeat with RubinsteinTaybi syndrome. 


\section{STAR*METHODS}

\section{KEY RESOURCES TABLE}

\begin{tabular}{|c|c|c|}
\hline REAGENT or RESOURCE & SOURCE & IDENTIFIER \\
\hline \multicolumn{3}{|l|}{ Antibodies } \\
\hline Rabbit anti-AUTS2 & (Gao et al., 2014) & $\mathrm{N} / \mathrm{A}$ \\
\hline Rabbit anti-PCGF3 & Reinberg lab & $\mathrm{N} / \mathrm{A}$ \\
\hline Rabbit anti-Ring1B & Bethyl Laboratories & $\begin{array}{l}\text { Cat\# A302-869A, } \\
\text { RRID:AB_1063277 } \\
3\end{array}$ \\
\hline Rabbit anti-RYBP & Sigma & $\begin{array}{ll}\text { Cat\# } & \text { PRS2227, } \\
\text { RRID:AB_1847589 }\end{array}$ \\
\hline Rabbit anti-NRF1 & Abcam & $\begin{array}{ll}\text { Cat\# } & \text { ab34682, } \\
\text { RRID:AB_2236220 }\end{array}$ \\
\hline Mouse anti-NRF1 & Abcam & $\begin{array}{ll}\text { Cat\# } & \text { ab55744, } \\
\text { RRID:AB_2154534 }\end{array}$ \\
\hline Mouse anti-P300 & Active motif & $\begin{array}{lr}\text { Cat\# } & 61401, \\
\text { RRID:AB_2716754 }\end{array}$ \\
\hline Rabbit anti-CBP & $\begin{array}{l}\text { Cell Signaling } \\
\text { Technology }\end{array}$ & $\begin{array}{lr}\text { Cat\# } & 7389, \\
\text { RRID:AB_2616020 }\end{array}$ \\
\hline Goat anti-Oct4 & $\begin{array}{ll}\text { Santa } & \text { Cruz } \\
\text { Biotechnology } & \\
\end{array}$ & $\begin{array}{lr}\text { Cat\# } & \text { sc-8628, } \\
\text { RRID:AB_653551 }\end{array}$ \\
\hline Mouse anti-HB9 & $\begin{array}{l}\text { Developmental } \\
\text { Studies Hybridoma } \\
\text { Bank }\end{array}$ & $\begin{array}{lr}\text { Cat\# } & 81.5 C 10, \\
\text { RRID:AB_2145209 }\end{array}$ \\
\hline Rabbit anti-H3 & Abcam & $\begin{array}{l}\text { Cat\# ab1791, } \\
\text { RRID:AB_302613 }\end{array}$ \\
\hline Rabbit anti-H2AK119ub1 & $\begin{array}{l}\text { Cell Signaling } \\
\text { Technology }\end{array}$ & $\begin{array}{l}\text { Cat\# 8240; RRID: } \\
\text { AB_10891618 }\end{array}$ \\
\hline Rabbit anti-H3K27me3 & $\begin{array}{l}\text { Cell Signaling } \\
\text { Technology }\end{array}$ & $\begin{array}{l}\text { Cat\# 9733; RRID: } \\
A B \_2616029\end{array}$ \\
\hline Rabbit anti-H3K27ac & Abcam & $\begin{array}{lr}\text { Cat\# } & \text { ab4729; } \\
\text { RRID: } & \text { AB_2118291 }\end{array}$ \\
\hline Rabbit anti-H3K4me3 & Abcam & $\begin{array}{l}\text { Cat\# ab8580; } \\
\text { RRID: AB_306649 }\end{array}$ \\
\hline Rabbit anti-Flag & Proteintech & $\begin{array}{l}\text { Cat\# 20543-1-AP; } \\
\text { RRID: } \\
\text { AB_11232216 }\end{array}$ \\
\hline Rabbit anti-Vinculin & $\begin{array}{l}\text { Cell Signaling } \\
\text { Technology }\end{array}$ & $\begin{array}{l}\text { Cat\# 13901; RRID: } \\
\text { AB_2728768 }\end{array}$ \\
\hline Rabbit anti-GAPDH & $\begin{array}{l}\text { Cell Signaling } \\
\text { Technology }\end{array}$ & $\begin{array}{l}\text { Cat\# 5174; RRID: } \\
A B \_10622025\end{array}$ \\
\hline
\end{tabular}




\begin{tabular}{|c|c|c|}
\hline Rabbit anti-Drosophila-specific $\mathrm{H} 2 \mathrm{Av}$ & Active motif & $\begin{array}{l}\text { Cat\# } 39715, \\
\text { RRID:AB_2793318 }\end{array}$ \\
\hline \multicolumn{3}{|l|}{ Bacterial and Virus Strains } \\
\hline Rosetta (DE3) Competent Cells & Novagen & Cat\# 70954 \\
\hline One-Shot Stbl3 chemically competent cells & Invitrogen & Cat\# C7373-03 \\
\hline DH10Bac & $\begin{array}{ll}\text { Thermo } & \text { Fisher } \\
\text { Scientific } & \end{array}$ & Cat\# 10361012 \\
\hline \multicolumn{3}{|l|}{ Chemicals, Peptides, and Recombinant Proteins } \\
\hline Lipofectamine 2000 & $\begin{array}{ll}\text { Thermo } & \text { Fisher } \\
\text { Scientific } & \\
\end{array}$ & Cat\# 11668027 \\
\hline Retinoic Acid & Sigma & Cat\# R2625 \\
\hline Smoothened Agonist, SAG & EMD/Calbiochem & Cat\# 566660 \\
\hline Anti-FLAG M2 affinity gel & Sigma & Cat\# A2220 \\
\hline FLAG ${ }^{\circledR}$ Peptide & Sigma & Cat\# F3290 \\
\hline Leukemia inhibitory factor & Reinberg lab & $\mathrm{N} / \mathrm{A}$ \\
\hline CHIR99021 & Tocris & Cat\# 4423 \\
\hline PD0325901 & Sigma & Cat\# PZ0162 \\
\hline Dynabeads protein $\mathrm{G}$ beads & $\begin{array}{ll}\text { Thermo } & \text { Fisher } \\
\text { Scientific } & \\
\end{array}$ & Cat\# 10003D \\
\hline SYBR-Gold Nucleic Acid Gel Stain & $\begin{array}{ll}\text { Thermo } & \text { Fisher } \\
\text { Scientific } & \\
\end{array}$ & Cat\# S11494 \\
\hline Agencourt AMPure XP Beads & Beckman Coulter & Cat\# A63882 \\
\hline RNase Inhibitor & NEB & Cat\# M0314L \\
\hline Poly Ethylene Glycol (PEG) 8000 & Sigma & $\begin{array}{l}\text { Cat\# 89510-250G- } \\
\text { F }\end{array}$ \\
\hline GTP & $\begin{array}{ll}\text { Thermo } & \text { Fisher } \\
\text { Scientific } & \\
\end{array}$ & Cat\# R1461 \\
\hline Calcein_AM & $\begin{array}{ll}\text { Thermo } & \text { Fisher } \\
\text { Scientific } & \\
\end{array}$ & Cat\# C1430 \\
\hline Ethidium homodimer-1 & Sigma & Cat\# 46043-1MG-F \\
\hline \multicolumn{3}{|l|}{ Critical Commercial Assays } \\
\hline Agilent High Sensitivity DNA Kit & Agilent Technologies & Cat\# 5067-4626 \\
\hline Nextera XT DNA Library Preparation Kit & illumina & Cat\# FC-131-1024 \\
\hline Maxima H Minus Reverse Transcriptase & Thermo Fisher & Cat\# EP0751 \\
\hline KAPA HiFi Hotstart PCR kit & Roche & Cat\# KK2502 \\
\hline
\end{tabular}




\begin{tabular}{|c|c|c|}
\hline Phusion High-Fidelity DNA Polymerase & NEB & Cat\# M0530L \\
\hline Quant-iT ${ }^{\mathrm{TM}}$ PicoGreen ${ }^{\mathrm{TM}}$ dsDNA Assay Kit & Thermo Fisher & Cat\# P7589 \\
\hline RNEasy plus Mini Kit & QIAGEN & Cat\# 74134 \\
\hline MinElute PCR Purification Kit & QIAGEN & Cat\# 28006 \\
\hline BCA Protein Assay Kit & Thermo Scientific & Cat\# 23225 \\
\hline End-It ${ }^{\mathrm{TM}}$ DNA End-Repair Kit & Lucigen & ER81050 \\
\hline Klenow Fragment $\left(3^{\prime} \rightarrow 5^{\prime}\right.$ exo- $)$ & NEB & $\mathrm{M} 0212 \mathrm{~L}$ \\
\hline T4 DNA Ligase (Rapid) & QIAGEN & $50-305-905$ \\
\hline $\mathrm{Q}^{\circledR}{ }^{\circledR}$ High-Fidelity DNA Polymerase & NEB & M0491L \\
\hline \multicolumn{3}{|l|}{ Deposited Data } \\
\hline Mouse brain AUTS2, NRF1, RYBP, PCGF3 ChIP-Seq & This study & GEO \\
\hline E14_ESC AUTS2, NRF1 ChIP-Seq & This study & GEO \\
\hline $\begin{array}{l}\text { Motor neuron AUTS2, NRF1, Ring1B, RYBP, } \\
\text { H2AK119ub, H3K27me3, and H3K27ac ChIP-Seq }\end{array}$ & This study & GEO \\
\hline Mouse brain, E14_ESC and Motor neuron RNA-Seq & This study & GEO \\
\hline Motor neuron scRNA-Seq & This study & GEO \\
\hline $\begin{array}{l}\text { Mouse brain input, AUTS2, P300, H2AK119ub, } \\
\text { H3K27me3, H3K27ac, H3K4me3, Ring1B and Pol II } \\
\text { ChIP-Seq }\end{array}$ & (Gao et al., 2014) & GEO: GSE60411 \\
\hline E14_ESC input, Pol II ChIP-Seq & (LeRoy et al., 2019) & GEO: GSE117155 \\
\hline $\begin{array}{l}\text { Motor neuron Pol II, H2AK119ub, H3K4me3, } \\
\text { H3K27me3 ChIP-Seq }\end{array}$ & $\begin{array}{l}\text { (Narendra et al., } \\
\text { 2015) }\end{array}$ & GEO: GSE60240 \\
\hline \multicolumn{3}{|l|}{ Experimental Models: Cell Lines } \\
\hline Human: HEK293 T-REx & Reinberg lab & $\mathrm{N} / \mathrm{A}$ \\
\hline Human: HEK293T 5XGal4TK-LuC & Reinberg lab & $\mathrm{N} / \mathrm{A}$ \\
\hline Mouse: ES cell line E14 & Reinberg lab & $\mathrm{N} / \mathrm{A}$ \\
\hline Mouse: ES cell line E14 Auts2 -/- & This study & $\mathrm{N} / \mathrm{A}$ \\
\hline Mouse: ES cell line E14 Nrf1 -/- & This study & $\mathrm{N} / \mathrm{A}$ \\
\hline Mouse: ES cell line E14 Auts2 T534P & This study & $\mathrm{N} / \mathrm{A}$ \\
\hline Mouse: ES cell line E14 Auts2 535-542 aa deletion & This study & $\mathrm{N} / \mathrm{A}$ \\
\hline \multicolumn{3}{|l|}{ Experimental Models: Organisms/Strains } \\
\hline Mouse: C57BL/6J & Jackson Laboratory & Strain 000664 \\
\hline Mouse: Nrf1-CKO & (Kiyama et al., 2018) & N/A \\
\hline Mouse: Nrf1-LacZ & (Kiyama et al., 2018) & N/A \\
\hline Mouse: Tbr1-TauGFP-IRESCreERT2 & (Kiyama et al., 2019) & $\mathrm{N} / \mathrm{A}$ \\
\hline Mouse: Pou4f1-CKOAP & (Badea et al., 2009) & Strain 010558 \\
\hline
\end{tabular}




\begin{tabular}{|c|c|c|}
\hline \multicolumn{3}{|l|}{ Oligonucleotides } \\
\hline $\begin{array}{l}\text { SgRNA and ssODN for Auts2, Nrf1 knockout, Auts } 2 \\
\text { HX mutant knock-in }\end{array}$ & This study & $\begin{array}{l}\text { See } \\
\text { Supplementary } \\
\text { Table } 2\end{array}$ \\
\hline Oligonucleotides, Barcode sequence for scRNA-Seq & This study & $\begin{array}{l}\text { See } \\
\text { Supplementary } \\
\text { Table } 3\end{array}$ \\
\hline \multicolumn{3}{|l|}{ Recombinant DNA } \\
\hline pSpCas9(BB)-2A-GFP & Addgene & Cat \# 48138 \\
\hline pSpCas9(BB)-2A-GFP-Auts2-KO-sgRNA & This study & N/A \\
\hline pSpCas9(BB)-2A-GFP-Auts2-T534P-sgRNA & This study & $\mathrm{N} / \mathrm{A}$ \\
\hline pSpCas9(BB)-2A-GFP-Auts2-535-542-del-sgRNA & This study & N/A \\
\hline pSpCas9(BB)-2A-GFP-Nrf1-KO-sgRNA & This study & $\mathrm{N} / \mathrm{A}$ \\
\hline pINTO-NFH(empty vector) & Reinberg lab & N/A \\
\hline pINTO-NFH-AUTS2-P517L & This study & N/A \\
\hline pINTO-NFH-AUTS2-T534P & This study & $\mathrm{N} / \mathrm{A}$ \\
\hline pINTO-NFH-AUTS2-535-542 del & This study & N/A \\
\hline pINTO-Gal4(empty vector) & Reinberg lab & N/A \\
\hline pINTO-Gal4-AUTS2-T534P & This study & N/A \\
\hline pINTO-Gal4-AUTS2-535-542 del & This study & $\mathrm{N} / \mathrm{A}$ \\
\hline \multicolumn{3}{|l|}{ Software and Algorithms } \\
\hline Bowtie2 v2.4.1 & $\begin{array}{ll}\text { (Langmead } & \text { and } \\
\text { Salzberg, 2012) } & \end{array}$ & $\begin{array}{l}\text { https://github.com/ } \\
\text { BenLangmead/bowti } \\
\underline{\text { e2 }}\end{array}$ \\
\hline STAR v2.6.1d & (Dobin et al., 2013) & $\begin{array}{l}\text { https://github.com/ } \\
\text { alexdobin/STAR }\end{array}$ \\
\hline MAC2 v2.1.1 & (Zhang et al., 2008) & $\begin{array}{l}\text { https://github.com/ } \\
\text { macs3- } \\
\text { project/MACS }\end{array}$ \\
\hline GSEA & $\begin{array}{l}\text { (Subramanian et al., } \\
\text { 2005) }\end{array}$ & $\begin{array}{l}\text { http://software.broa } \\
\text { dinstitute.org/gsea/i } \\
\text { ndex.jsp }\end{array}$ \\
\hline fgsea & $\begin{array}{l}\text { (Korotkevich et al., } \\
\text { 2019) }\end{array}$ & $\begin{array}{l}\text { https://github.com/ } \\
\text { ctlab/fgsea }\end{array}$ \\
\hline BEDTools v2.27.1 & $\begin{array}{l}\text { (Quinlan and Hall, } \\
\text { 2010) }\end{array}$ & $\begin{array}{l}\text { https://github.com/ } \\
\text { arq5x/bedtools }\end{array}$ \\
\hline
\end{tabular}




\begin{tabular}{|c|c|c|}
\hline Picard v2.18.11 & Broad Institute & $\begin{array}{l}\frac{\text { https://github.com/ }}{\text { broadinstitute/picar }} \\
\underline{\text { d }}\end{array}$ \\
\hline HOMER v4.10 & (Heinz et al., 2010) & $\begin{array}{l}\text { http://homer.ucsd.e } \\
\mathrm{du} /\end{array}$ \\
\hline deepTools v3.2.1 & $\begin{array}{l}\text { (Ramírez et al., } \\
\text { 2016) }\end{array}$ & $\begin{array}{l}\underline{\text { https://github.com/ }} \\
\underline{\text { deeptools/deepTool }} \\
\underline{\text { s }}\end{array}$ \\
\hline Samtools v1.9 & (Li et al., 2009) & $\begin{array}{l}\text { https://github.com/ } \\
\text { samtools/ }\end{array}$ \\
\hline ChIPseeker v1.8.6 & (Yu et al., 2015) & $\begin{array}{l}\text { https://github.com/ } \\
\text { YuLab- } \\
\text { SMU/ChIPseeker }\end{array}$ \\
\hline Integrative Genomics Viewer (IGV) & Broad Institute & $\frac{\text { http://www.broadin }}{\text { stitute.org/igv/ }}$ \\
\hline Prism & GraphPad Software & $\begin{array}{l}\text { https://www.graphp } \\
\text { ad.com/scientific- } \\
\text { software/prism/ }\end{array}$ \\
\hline Benchling & Benchling.com & $\begin{array}{l}\text { https://www.benchli } \\
\text { ng.com/ }\end{array}$ \\
\hline SRA Toolkit & $\mathrm{NCBI}$ & $\begin{array}{l}\text { https://github.com/ } \\
\text { ncbi/sra-tools }\end{array}$ \\
\hline RStudio & RStudio & https://rstudio.com/ \\
\hline R V4.0.0 & R-project & $\begin{array}{l}\text { https://www.r- } \\
\text { project.org/ }\end{array}$ \\
\hline featureCounts v1.6.3 & (Liao et al., 2014) & $\begin{array}{l}\text { https://github.com/t } \\
\text { orkian/subread- } \\
\text { 1.6.1 }\end{array}$ \\
\hline ComplexHeatmap & (Gu et al., 2016) & $\begin{array}{l}\text { https://github.com/i } \\
\text { okergoo/ComplexHe } \\
\text { atmap }\end{array}$ \\
\hline Seurat v3.1.4 & (Butler et al., 2018) & $\begin{array}{l}\text { https://github.com/ } \\
\text { satijalab/seurat }\end{array}$ \\
\hline zUMIs v2.9.4 & (Parekh et al., 2018) & $\begin{array}{l}\text { https://github.com/ } \\
\text { sdparekh/zUMIs }\end{array}$ \\
\hline Presto & $\begin{array}{l}\text { (Korsunsky et al., } \\
2019)\end{array}$ & $\begin{array}{l}\text { https://github.com/i } \\
\text { mmunogenomics/pr } \\
\text { esto }\end{array}$ \\
\hline & & \\
\hline
\end{tabular}




\section{Lead Contact and Materials Availability}

Please direct inquiry to the Lead Contact, Danny Reinberg (danny.reinberg@nyumc.org). All unique/stable reagents generated in this study are available from the Lead Contact with a completed Materials Transfer Agreement.

\section{Experimental Model and Subject Details}

\section{Animals}

All mice were housed with a 12 hour light-dark cycle. Mixed cohorts of female and male mice were used for all experiments to minimize gender effects. All animal procedures followed the US Public Health Service Policy on Humane Care and Use of Laboratory Animals and were approved by the Animal Welfare Committee at New York University and the University of Texas McGovern Medical School at Houston.

\section{Cell lines and culture condition}

All ESC lines (E14 and derivatives) were grown in DMEM supplemented with 15\% FBS, Lglutamine, penicillin/streptomycin, sodium pyruvate, non-essential amino acids, $0.1 \mathrm{mM} \beta$ mercaptoethanol, LIF, and $2 \mathrm{i}$ inhibitors, which include $1 \mu \mathrm{M}$ MEK1/2 inhibitor (PD0325901) and $3 \mu \mathrm{M}$ GSK3 inhibitor (CHIR99021) on $0.1 \%$ gelatin coated plates.

HEK293 T-Rex and HEK293T 5XGal4TK-Luc cells were cultured in standard DMEM supplemented with $10 \% \mathrm{FBS}, 100 \mathrm{U} / \mathrm{mL}$ penicillin-streptomycin.

WT and mutant NFH-AUTS2 inducible cell lines were obtained by transfecting each pINTO-NFH plasmid into 293 T-Rex cells, and WT and mutant Gal4-AUTS2 inducible cell lines were obtained by transfecting each pINTO-Gal4 plasmid into HEK293T 5XGal4TK-Luc cells. Transfected cells were seeded at limiting dilutions, and isolated clones were screened by western blot.

\section{Clinical Cohort}

The initial proband (LR05-007) was identified through trio-based exome sequencing in a cohort of 100 individuals with cerebellar malformations(Aldinger et al., 2019). We recruited 6 additional individuals with de novo pathogenic or likely pathogenic variants in AUTS2 by sharing data through GeneMatcher(Sobreira et al., 2015) or through collaboration with colleagues. The 7 individuals in this cohort ( 2 females, 5 males) were 1 to 15.5 years of age at the time of their most recent evaluation. We obtained clinical data for all patients, including features tabulated in a reported AUTS2 clinical severity score(Beunders et al., 2013, 2015, 2016). Written informed consent was obtained from all participants through protocols approved by Institutional Review Boards at the local institution or at Seattle Children's Hospital. 


\section{METHOD DETAILS}

\section{Immunoprecipitation and Proteomics}

Cell pellets were prepared from cell culture plates or mouse brain. Nuclei were extracted using HMSD buffer (20 mM HEPES, pH 7.5 at $4^{\circ} \mathrm{C}, 5 \mathrm{mM} \mathrm{MgCl}_{2}, 250 \mathrm{mM}$ sucrose, $25 \mathrm{mM} \mathrm{NaCl}, 1 \mathrm{mM}$ DTT) supplemented with protease inhibitors $(0.2 \mathrm{mM}$ PMSF, $1 \mu \mathrm{g} / \mathrm{mL}$ Pepstatin $\mathrm{A}, 1 \mu \mathrm{g} / \mathrm{mL}$ Leupeptin, and $1 \mu \mathrm{g} / \mathrm{mL}$ Aprotinin) and phosphatase inhibitors (10 $\mathrm{mM} \mathrm{NaF}$ and $1 \mathrm{mM} \mathrm{Na}_{3} \mathrm{VO}_{4}$ ), and incubated on ice for $5 \mathrm{~min}$. Lysates were pelleted at 3,000 rpm for $5 \mathrm{~min}$ at $4^{\circ} \mathrm{C}$ and nuclei pellets were washed one more time with HMSD buffer. The resulting nuclei pellets were resuspended in $\mathrm{BC} 420$ high salt buffer $\left(20 \mathrm{mM}\right.$ Tris- $\mathrm{HCl}$ at $\mathrm{pH} 7.9,1.5 \mathrm{mM} \mathrm{MgCl}_{2}, 0.42 \mathrm{M} \mathrm{NaCl}$, $0.5 \mathrm{mM}$ DTT, $0.2 \mathrm{mM}$ EDTA) supplemented with protease inhibitors and phosphatase inhibitors for lysing at $4^{\circ} \mathrm{C}$ for $1 \mathrm{hr}$ with occasional pipetting. Lysates were then pelleted at $20,000 \times \mathrm{g}$ for $15 \mathrm{~min}$ at $4^{\circ} \mathrm{C}$. Finally, supernatants were collected and subjected to dialysis in Buffer D ( $20 \mathrm{mM}$ HEPES, $150 \mathrm{mM} \mathrm{NaCl}, 1.5 \mathrm{mM} \mathrm{MgCl}, 0.2 \mathrm{mM}$ EDTA, and $5 \%$ glycerol) overnight at $4^{\circ} \mathrm{C}$. Prior to any subsequent applications, nuclear extracts were centrifuged again at 20,000 g for $10 \mathrm{~min}$ at $4^{\circ} \mathrm{C}$ to remove any precipitate. Supernatants were collected, and protein concentrations were quantified via bicinchonic acid (BCA) assay. For immunoprecipitation, 1-2 mg of nuclear extract was incubated with $1 \sim 3 \mu \mathrm{g}$ of antibody. After incubation at $4^{\circ} \mathrm{C}$ for $2 \mathrm{hr}, 30 \mathrm{ul}$ of protein G beads were added for incubation at $4^{\circ} \mathrm{C}$ overnight. Beads were washed three times with Buffer D (20 mM HEPES, $150 \mathrm{mM} \mathrm{NaCl}, 1.5 \mathrm{mM} \mathrm{MgCl}_{2}, 0.2 \mathrm{mM}$ EDTA, and 5\% glycerol), and eluted with $0.2 \mathrm{M}$ glycine $(\mathrm{pH} 2.6)$ or $1 \times$ SDS loading buffer. Proteins from immunoprecipitation were separated by SDS-PAGE, using 4\%-12\% NuPAGE Novex Bis-Tris gels and then stained with Coomassie Blue. Bands were excised from gels and digested with trypsin, followed by standard LC-MS/MS procedure.

\section{Whole cell extract and western blotting}

Cells were harvested and lysed with CHAPS-Urea buffer $(50 \mathrm{mM}$ Tris-HCl, pH 7.9, 8M Urea, and $1 \%$ CHAPS) containing protease inhibitors and phosphatase inhibitors as mentioned above. The cell suspension was briefly sonicated ( $40 \%$ amplitude, 5 strokes) and centrifuged at 20,000 $\mathrm{g}$ at $4^{\circ} \mathrm{C}$ for $20 \mathrm{~min}$. The supernatant was collected and protein concentrations were quantified via bicinchonic acid (BCA) assay. Proteins were separated using a 6\%-12\% SDS PAGE gel, and transferred onto a PVDF membrane. Membranes were blocked with 5\% milk in PBST at RT for 1 $\mathrm{hr}$ and incubated with primary antibody overnight at $4^{\circ} \mathrm{C}$. Membranes were washed 3 times with PBST and then incubated with HRP-conjugated secondary antibodies for $1 \mathrm{hr}$ at RT, followed by exposure.

\section{CRISPR-mediated genome editing}

To generate stable Auts2 and Nrf1 KO cell lines, sgRNAs were designed using CRISPR design tool in https://benchling.com. sgRNAs in Table S1 were cloned in pSpCas9(BB)-2A-GFP (PX458, a gift from Feng Zhang, Addgene plasmid \#48138) and transfected into $\mathrm{mESCs}$, using Lipofectamine 2000 (Life Technologies). GFP-positive cells were sorted $48 \mathrm{hr}$ after transfection and 20,000 cells were plated on a $15 \mathrm{~cm}$ dish. Single mESC was allowed to grow to a colony for $\sim 5$ days and then was picked, trypsinized in Accutase for $5 \mathrm{~min}$, and split into two individual wells of two 96well plates for genotyping and culture, respectively. Genomic DNA was extracted using lysis buffer (50 mM Tris- $\mathrm{HCl}$, pH 8, $2 \mathrm{mM} \mathrm{NaCl}, 10 \mathrm{mM}$ EDTA, 0.1\% SDS) supplemented with protease 
$\mathrm{K}$, and genotyping PCRs were performed using primers (Table S2) surrounding the target site. The resulting PCR products were sent for sequencing to determine the presence of a deletion or a mutation event. Clones were further confirmed by western blot.

For endogenously knock-in the mutations in AUTS2 HX repeat in mESC, a SSODN donor used for homology directed repair and CRISPR/Cas9 plasmid ( $p \times 458$ ) with designed sgRNA (see Table S2 also) were co-transfected into mESCs, using Lipofectamine 2000. The following FACS, colony picking and characterization are the same as generating $\mathrm{KO}$ lines described above.

\section{Motor Neuron Differentiation}

E14 mouse embryonic stem cells (mESCs) were cultured in standard medium supplemented with LIF, and $2 \mathrm{i}$ conditions as described above. For motor neuron (MN) differentiation, the previously described protocol was applied(Narendra et al., 2015). Briefly, about 4 million mESCs were plated in a $500 \mathrm{~cm}^{2}$ square dish and differentiated into embryoid bodies in AK medium (250 ml advanced DMEM/F12, $250 \mathrm{ml}$ neurobasal medium, $75 \mathrm{ml}$ knockout serum, L-glutamine, penicillin/streptomycin, $0.1 \mathrm{mM} \beta$-mercaptoethanol) for 2 days. Embryoid bodies were then diluted by $1: 4$ and further patterning was induced by freshly adding $1 \mu \mathrm{M}$ all-trans-retinoic acid (RA) and $0.5 \mu \mathrm{M}$ smoothened agonist (SAG) for an additional 4 days. Fresh medium was added after 2 days to support motor neuron survival.

\section{Luciferase reporter assay}

HEK293T 5XGal4 TK-Luc cells stably transfected with pINTO-GAL4 vector control or with inserts of interest were treated with $100 \mathrm{ng} / \mathrm{ml}$ doxycycline. Cells were lysed by adding $250 \mathrm{ul}$ of icecold lysis buffer (0.2\% Triton X-100, $100 \mathrm{mM}$ potassium phosphate, $\mathrm{pH} 7.8$, and $1 \mathrm{mM}$ DTT) and shaking for $10 \mathrm{~min}$ at $4^{\circ} \mathrm{C}$. The cell lysate was centrifuged at $20,000 \mathrm{~g}$ for $10 \mathrm{~min}$ and the protein concentration of the resulting supernatant was determined by bicinchonic acid (BCA) assay. 30 ug of the supernatant was assayed for luciferase activity using luciferase assay substrate (Promega).

\section{X-gal staining}

Mouse brain was fixed by perfusion with $10 \%$ neutral buffered formalin. Extracted brain was embedded in OCT compound then sectioned into 50 um thickness. Sections were dried at RT for $3 \mathrm{hr}$ and then washed with wash buffer $\left(0.1 \mathrm{M}\right.$ sodium phosphate containing $2 \mathrm{mM} \mathrm{MgCl}_{2}$, $0.01 \%$ deoxycholate, and $0.02 \%$ Nonidet P-40, pH 7.3). LacZ color reaction was performed in wash buffer containing $5 \mathrm{mM}$ potassium ferrocyanide, $5 \mathrm{mM}$ potassium ferricyanide, and 1 $\mathrm{mg} / \mathrm{ml} \mathrm{X}$-gal at $37^{\circ} \mathrm{C}$ overnight. Color reaction was terminated by incubation in $10 \%$ formalin for 10 min. Post-fixed sections were washed, dehydrated, and mounted with Cytoseal 60 (Thermo Fisher Scientific). Images were collected with a Canon EOS 10 digital camera (Melville, NY) mounted on an Olympus IX71 microscope.

\section{Immuno-histochemical analysis}

Mouse brain was fixed by perfusion with $10 \%$ neutral buffered formalin. Extracted brain was embedded in OCT compound, and then sectioned into 100 um thickness. Sections were 
incubated with anti-GFP (1:1000, Invitrogen) antibody. Alexa-488 conjugated secondary antibody was used in 1:800 dilution (Jackson Immuno-reserach).

\section{Alkaline phosphatase (AP) staining}

Tbr1 ${ }^{\text {CreERT2/+ }}: \mathrm{Nrf1}^{f x /+}:$ Pou $4 f 1^{\text {CKO/+ }}$ and Tbr1 ${ }^{\text {CreERT2/+ }}: N r f 1^{f x / f x}: P o u 4 f 1^{\text {CKO/+ }}$ mice were used for AP staining 3 months after tamoxifen injection. Brains were fixed by perfusion with $10 \%$ neutral buffered formalin. Fixed brain was embedded in OCT compound and sectioned into 100 um thickness. Retinal flat-mounts were fixed in $10 \%$ neutral buffered formalin for 10 min at RT. Brain sections and retinas were incubated in heated PBS for $30 \mathrm{~min}$ in a $65^{\circ} \mathrm{C}$ water bath to inactivate endogenous AP activity. AP color reaction was performed in $0.1 \mathrm{M}$ Tris, $\mathrm{pH} 9.5,0.1 \mathrm{M}$ $\mathrm{NaCl}, 50 \mathrm{mM} \mathrm{MgCl}, 0.34 \mathrm{~g} / \mathrm{ml}$ nitroblue tetrazolium and $0.175 \mathrm{~g} / \mathrm{ml}$ 5-bromo-4-chrolo-3indolyl-phosphate for overnight at RT. Stained tissues were washed three times in PBS, postfixed with $10 \%$ neutral buffered formalin, dehydrated with a series of ethanol, then cleared with 2:1 benzyl benzoate/benzyl alcohol. Tiled images were collected using a Zeiss Axio Imager 2 microscope (Carl Zeiss).

\section{ChIP-seq library preparation}

For cross-linking, ESCs were fixed in 1\% formaldehyde for $10 \mathrm{~min}$ at RT directly on plates and quenched with $125 \mathrm{mM}$ glycine for $5 \mathrm{~min}$ at RT. For cross-linking of MN, ESC-derived motor neuron cultured for 6 days were dissociated with $0.05 \%$ trypsin, neutralized, fixed in $1 \%$ formaldehyde for $15 \mathrm{~min}$ at RT and then quenched with $125 \mathrm{mM}$ glycine for $5 \mathrm{~min}$ at RT. For cross-linking of mouse brain, mouse whole brains were quickly dissected at postnatal day one and homogenized with a glass douncing homogenizer using first a loose, then a tight pestle. The cell homogenate was fixed with a final concentration of $1 \%$ formaldehyde for $10 \mathrm{~min}$ at RT and the reaction was quenched with $0.125 \mathrm{M}$ glycine for $5 \mathrm{~min}$ at RT.

Cell pellets were washed twice in PBS and nuclei were isolated using buffers in the following order: LB1 (50 mM HEPES, pH 7.5 at $4^{\circ} \mathrm{C}, 140 \mathrm{mM} \mathrm{NaCl}, 1 \mathrm{mM}$ EDTA, 10\% Glycerol, 0.5\% NP40, $0.25 \%$ Triton $\mathrm{X} ; 10 \mathrm{~min}$ at $\left.4^{\circ} \mathrm{C}\right)$, LB2 $\left(10 \mathrm{mM}\right.$ Tris, $\mathrm{pH} 8$ at $4^{\circ} \mathrm{C}, 200 \mathrm{mM} \mathrm{NaCl}, 1 \mathrm{mM}$ EDTA, 0.5 $\mathrm{mM}$ EGTA; $10 \mathrm{~min}$ at $4^{\circ} \mathrm{C}$ ), and LB3 (10 mM Tris, pH 7.5 at $4^{\circ} \mathrm{C}, 1 \mathrm{mM}$ EDTA, $0.5 \mathrm{mM}$ EGTA, and $0.5 \% \mathrm{~N}$-Lauroylsarcosine sodium salt). Chromatin was fragmented to an average size of $250 \mathrm{bp}$ in LB3 buffer using a Diagenode Bioruptor. $200 \mu \mathrm{g}$ sonicated chromatin, 4 ug antibody and $20 \mathrm{ul}$ Dynabeads were used in each ChIP reaction supplemented with $0.5 x$ volumn of incubation buffer (3\% Triton X, 0.3\% Na Deoxycholate, 15 mM EDTA). $1 \mu \mathrm{g}$ of Drosophila chromatin and 0.2 $\mu \mathrm{g}$ of anti-Drosophila H2A.X antibody were added in each ChIP reaction as spike-in references, except $3 \mu \mathrm{g}$ of Drosophila chromatin was used for H3K27ac and H2AK119ub ChIP. After 5 consecutive washes with RIPA buffer (50 mM HEPES, pH 7.5 at $4^{\circ} \mathrm{C}, 0.7 \%$ Na Deoxycholate, 1 mM EDTA, 1\% NP40, $500 \mathrm{mM} \mathrm{LiCl}$ ) and one wash with TE $+50 \mathrm{mM} \mathrm{NaCl}$, the beads-bound DNA was eluted in freshly prepared elution buffer (50 mM Tris, pH 8, $10 \mathrm{mM}$ EDTA, $1 \%$ SDS) at $65^{\circ} \mathrm{C}$ for 20 min. Eluted DNA was de-crosslinked at $65^{\circ} \mathrm{C}$ overnight, followed by protease $\mathrm{K}$ and RNase A treatment.

For Library preparation, IP'ed DNA ( 1-30 ng) was end-repaired using End-It Repair Kit, tailed with deoxyadenine using Klenow exo-, and ligated to custom adapters with T4 Rapid DNA Ligase (Enzymatics). Fragments of 200-600 bp were size-selected using Agencourt AMPure XP 
beads (0.5X and 0.3X), and subjected to PCR amplification using Q5 DNA polymerase. Libraries were size-selected using Agencourt AMPure XP beads $(0.75 \mathrm{X})$, quantified by Qubit ${ }^{\mathrm{TM}} \mathrm{dsDNA}$ HS Assay Kit and quality checked by High Sensitivity D1000 ScreenTape. Libraries were sequenced as $50 \mathrm{bp}$ single-end reads on the Illumina HiSeq 4000 platform.

\section{RNA-seq library preparation}

Total RNA was isolated with Tripure isolation reagent and gDNA was removed by RNeasy Plus mini kit. PolyA+ RNA was isolated from 5 ug total RNA using Dynabeads Oligo(dT) 25 , fragmented with $\mathrm{Mg}^{2+}$ contained in the $1^{\text {st }}$ strand buffer at $94^{\circ} \mathrm{C}$ for $15 \mathrm{~min}$, and reverse transcribed using Superscript III and random hexamers to synthesize the first strand cDNA. Single strand cDNA was precipitated and dTTP was removed by phenol/chloroform/isoamyl alcohol extraction and ethanol precipitation. Second strand CDNA was synthesized with dUTP to generate strand asymmetry using DNA Pol I and E. coli ligase, and then purified by MinElute PCR Purification Kit. Double-stranded DNA was end-repaired, A-tailed, and ligated to custom barcode adapters as described above. RNA-seq libraries were sequenced as $50 \mathrm{bp}$ paired-end reads on the Illumina HiSeq 4000 platform or NovaSeq 6000 platform.

\section{Single cell RNA-seq library preparation}

Single cell RNA-seq library by Smart-seq3 technique was generated according to the published protocol(Hagemann-Jensen, 2020; Hagemann-Jensen et al., 2020), with the following modifications. ESC-derived motor neuron cultured for 6 days were dissociated with $0.05 \%$ trypsin and stained with 0.2 uM Calcein AM and 8 uM Ethidium homodimer-1 at RT for 15 min. Single viable cells were sorted using Fluorescence Activated Cell Sorting to single wells of 96well fully-skirted Eppendorf PCR plates in 3 uL lysis buffer (5\% PEG8000, 0.1\% Triton X-100, 0.5 unit/ul RNAse Inhibitor, 0.5 uM OligodT $_{30} \mathrm{VN}, 0.5 \mathrm{mM}$ dNTP in nuclease free water). The plates were immediately covered, spun at $2000 \mathrm{rpm}$ for $1 \mathrm{~min}$ at $4^{\circ} \mathrm{C}$, and stored at $-80^{\circ} \mathrm{C}$ until further analysis. In each plate, well A1 was left empty and 100 cells were sorted to well $\mathrm{H} 1$ for quality control and they were excluded for downstream analysis.

Plates were incubated at $72^{\circ} \mathrm{C}$ for 10 ? min for lysing the cells and denaturing the RNA. Next, 1? $\mathrm{\mu l}$ of reverse transcription $\operatorname{mix}(25$ ? $\mathrm{mM}$ Tris- $\mathrm{HCl}, \mathrm{pH} 8,30$ ? $\mathrm{mM} \mathrm{NaCl}, 1$ ? $\mathrm{mM} \mathrm{GTP}, 2.5$ ? $\mathrm{mM}$ $\mathrm{MgCl}_{2}$, 8? $\mathrm{mM}$ DTT, 0.5 unit/ul RNAse Inhibitor, 2] $\mu \mathrm{M}$ Smart-seq3 TSO, 2? unit/ul Maxima Hminus reverse transcriptase enzyme) was added to each well for reverse transcription and template switching at $42^{\circ} \mathrm{C}$ for 90 ? min followed by 10 cycles at $50^{\circ} \mathrm{C}$ for 2 ? min and at $42^{\circ} \mathrm{C}$ for 2? ? min and the reaction was inactivated at $85^{\circ} \mathrm{C}$ for 5 ? min. cDNA pre-amplification was performed by adding 6? $\mu$ l of PCR mix (1×Kapa HiFi HotStart buffer, 0.02? unit/ul KAPA HiFi DNA polymerase, 0.5 [ $\mathrm{mM} \mathrm{MgCl}_{2}, 0.3$ ? $\mathrm{mM}$ dNTPS, 0.5? $\mu \mathrm{M}$ Smartseq3 forward PCR primer and 0.1? $\mu \mathrm{M}$ Smartseq3 reverse PCR primer) with the following protocol: 3 ? min at $98^{\circ} \mathrm{C}$ for initial denaturation, $18-24$ cycles of $20 \mathrm{~s}$ at $98^{\circ} \mathrm{C}$, 30? $\mathrm{s}$ at $65^{\circ} \mathrm{C}$ and 6 ? $\mathrm{min}$ at $72^{\circ} \mathrm{C}$, followed by a final elongation for 5 ? min at $72^{\circ} \mathrm{C}$. PCR cycles were determined for each cell type by prior experiments and 19 cycles were used for motor neuron to obtain 10 ng purified cDNA.

Pre-amplified cDNA was purified by $0.6 x$ volume of AMpure XP beads, eluted in 14 ul nuclease free water, quantified using the Quant-iT PicoGreen dsDNA Assay Kit and size distributions were 
checked on a high-sensitivity DNA chip. cDNA was then diluted to 200?pg/ $\mu$, and 1 ul was used for tagmentation by mixing with $1 \mu \mathrm{l}$ of tagmentation reaction mixture (10?mM TAPS, $\mathrm{pH} 8.5$, 5 [? $\mathrm{mM} \mathrm{MgCl}_{2}, 8 \% \mathrm{PEG} 8000,0.08 \mathrm{ul} \mathrm{Tn} 5 \mathrm{mix}$ (illumina)). The reaction was performed at $55^{\circ} \mathrm{C}$ for 7?min, followed by heat inactivation at $80^{\circ} \mathrm{C}$ for 5 ? min. Library amplification of the tagmented samples was performed using custom-designed index primers and by adding 5? $\mu$ of PCR mix (1× Phusion High-Fidelity buffer, 0.01?unit/ul Phusion High-Fidelity DNA Polymerase, 0.2? $\mathrm{mM}$ $\mathrm{dNTPS}, 0.1$ ? $\mu \mathrm{M}$ forward indexed primer and 0.1 ? $\mu \mathrm{M}$ reverse indexed primer). Amplification was

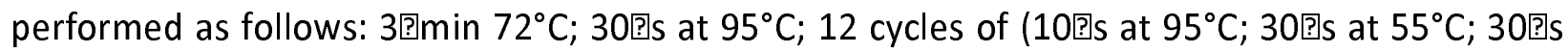
at $72^{\circ} \mathrm{C}$ ); and 5 ?min at $72^{\circ} \mathrm{C}$. Samples from one 96 -well plate were pooled, and then purified with $0.6 x$ volume of Ampure XP beads. Libraries were quantified by Qubit ${ }^{\mathrm{TM}}$ dsDNA HS Assay Kit and quality checked on a high-sensitivity DNA chip. The size averaged at $\sim 1 \mathrm{~Kb}$ should be expected. ScRNA-seq libraries were sequenced as $50 \mathrm{bp}$ paired-end reads on the Illumina NovaSeq 6000 platform and importantly, 20\% phiX spike-in was added to resolve the low complexity issue in the first 20 bps. All oligos used are listed in Table S3.

\section{QUANTIFICATION AND STATISTICAL ANALYSIS}

\section{RNA-seq data analysis}

Reads were aligned to the mouse reference genome mm10 using STAR with parameters: -outFilterMismatchNoverLmax 0.2 --outFilterMultimapNmax 1 --outSAMstrandField intronMotif --outSAMmapqUnique 60 --twopassMode Basic --outSJfilterReads Unique -outFilterIntronMotifs RemoveNoncanonical. Gene counts were calculated using featureCounts with parameters: - $p$-s 2 -t exon, and RefSeq mm10 annotation downloaded from GENCODE. The output gene count tables were used as input into DeSeq 2 for normalization and differential expression analysis. For comparing the expression level of different genes within a sample, TPM (transcripts per kilobase million) is calculated as: TPM $=($ CDS read count * mean read length * $\left.10^{6}\right) /($ CDS length * total transcript count)

\section{ChIP-seq data analysis}

Reads were aligned to the mouse reference genome mm10 and dm6 for spike-in samples, using Bowtie2 with default parameters. Reads of quality score less than 30 were removed using samtools and PCR duplicates were removed using picard. Regions in mm10 genome blacklist was removed using bedtools and bigwig files were generated using deeptools and parameters: -binSize 50 --normalizeUsing RPKM --ignoreDuplicates --ignoreForNormalization chrX -extendReads 250 for visualization in IGV. Peaks were called using MACS2 with parameters: - $g$ mm --keep-dup 1 --nomodel --extsize 300. Genomic peak annotation was performed with the R package ChIPseeker considering the region $\pm 3 \mathrm{~kb}$ around the TSS as the promoter. Peak overlapping analysis was performed using the Python package Intervene and visualized using the Python package Matplotlib. Motif discovery was performed using HOMER with default parameters.

For visualization of ChIP-seq, uniquely aligned reads mapping to the mouse genome were normalized using dm6 spike-in as described previously(Orlando et al., 2014). Heatmaps were 
performed using the functions computeMatrix followed by plotHeatmap and plotProfile from deepTools.

\section{scRNA-seq data analysis}

Raw non-demultiplexed fastq files were processed using zUMIs with STAR to generate expression profiles for both the $5^{\prime}$ ends containing UMIs as well as combined full-length and UMI data. The parameters: find pattern: ATTGCGCAATG, UMI (12-19), cDNA (23-50) were specified for Read 1 . The UMI count table containing both intron and exon reads was used by Seurat for downstream analysis. Cells with more than 3000 genes detected and less than $5 \%$ of reads mapping to mitochondrial genome were retained. Data were normalized (scTransform) and used for principal component analysis dimensionality reduction, followed by louvain clustering and UMAP dimensionality reduction. Major cell types were readily identifiable by common marker genes: posterior neural progenitor (PNP) expressing Sox3, posterior and ventral neural progenitor (PVNP) expressing Hoxd4, newborn motor neuron (NMN) expressing Neurog2, and motor neuron (MN) expressing $\mathrm{Mnx1}$ and Chat.

Differential gene expression analysis between defined cell clusters was performed using $R$ package presto. The top 500 DEGs were ordered by $p$ value. Averaged expression among the defined cell clusters was scaled by row and used as input to R package ComplexHeatmap for visualization.

\section{DATA AND CODE AVAILABILITY}

The accession numbers for the raw data FASTQ files and processed files for all sequencing data will be available on GEO. Original gel imaging data can be accessed from Mendeley: https://data.mendeley.com/datasets/69vsfxr2n6/draft?a=d996b718-00f6-40c6-9e1b-

\section{$\underline{879175 d f f 621}$}

\section{ACKNOWLEDGMENTS}

We thank Drs. Lynne Vales, Esteban Mazzoni, Anne Schaefer for critical reading of the manuscript as well as past and current Reinberg laboratory members for critical comments and discussions. We also thank the New York University Langone Medical Center (NYULMC) Genome Technology Center for help with sequencing, the NYULMC Cytometry and Cell Sorting Core for help with FACS, and the NYULMC Animal Facility for help with mice housing. This study utilized computing resources at the High-Performance Computing Facility of the Center for Health Informatics and Bioinformatics at the NYULMC. This work was supported by grants to D.R. from the NIH (R01NS100897, R01CA199652) and the Simon Foundation Grant \#240344 to D.R.) and the Howard Hughes Medical Institute.

\section{AUTHOR CONTRIBUTIONS}

S.L. and D.R. conceived the project, designed the experiments, and wrote the paper; S.L. performed most of the experiments and all the bioinformatic analysis; K.A.A., C.V.C., M.D. and H.K.M performed the clinical and genetic analyses under the supervision of W.B.D.; S.G.C., I.I., 
E.E., C.Z., O.Z., M.S., A.S.P., M.S.P., M.T.C., A.W., A.S. and L.G. collected the clinical data; T.K. performed the Nrf1 mouse studies under the supervision of T.C.B. and C.-A.M.; D.R. supervised the study.

\section{DECLARATION OF INTERESTS}

D.R. is a cofounder of Constellation and Fulcrum Pharmaceuticals. Other authors declare no competing interests.

\section{REFERENCES}

Ajmone, P.F., Avignone, S., Gervasini, C., Giacobbe, A., Monti, F., Costantino, A., Esposito, S., Marchisio, P., Triulzi, F., and Milani, D. (2018). Rubinstein-Taybi syndrome: New neuroradiological and neuropsychiatric insights from a multidisciplinary approach. Am J Med Genet B Neuropsychiatr Genet 177, 406-415.

Aldinger, K.A., Timms, A.E., Thomson, Z., Mirzaa, G.M., Bennett, J.T., Rosenberg, A.B., Roco, C.M., Hirano, M., Abidi, F., Haldipur, P., et al. (2019). Redefining the Etiologic Landscape of Cerebellar Malformations. The American Journal of Human Genetics 105, 606-615.

Aloia, L., Stefano, B.D., and Croce, L.D. (2013). Polycomb complexes in stem cells and embryonic development. Development 140, 2525-2534.

Badea, T.C., Cahill, H., Ecker, J., Hattar, S., and Nathans, J. (2009). Distinct Roles of Transcription Factors Brn3a and Brn3b in Controlling the Development, Morphology, and Function of Retinal Ganglion Cells. Neuron 61, 852-864.

Bannister, A.J., and Kouzarides, T. (1996). The CBP co-activator is a histone acetyltransferase. Nature 384, 641-643.

Becht, E., McInnes, L., Healy, J., Dutertre, C.-A., Kwok, I.W.H., Ng, L.G., Ginhoux, F., and Newell, E.W. (2019). Dimensionality reduction for visualizing single-cell data using UMAP. Nature Biotechnology 37, 38-44.

Bernstein, B.E., Mikkelsen, T.S., Xie, X., Kamal, M., Huebert, D.J., Cuff, J., Fry, B., Meissner, A., Wernig, M., Plath, K., et al. (2006). A Bivalent Chromatin Structure Marks Key Developmental Genes in Embryonic Stem Cells. Cell 125, 315-326.

Beunders, G., Voorhoeve, E., Golzio, C., Pardo, L.M., Rosenfeld, J.A., Talkowski, M.E., Simonic, I., Lionel, A.C., Vergult, S., Pyatt, R.E., et al. (2013). Exonic Deletions in AUTS2 Cause a Syndromic Form of Intellectual Disability and Suggest a Critical Role for the C Terminus. The American Journal of Human Genetics 92, 210-220.

Beunders, G., de Munnik, S.A., Van der Aa, N., Ceulemans, B., Voorhoeve, E., Groffen, A.J., Nillesen, W.M., Meijers-Heijboer, E.J., Frank Kooy, R., Yntema, H.G., et al. (2015). Two male 
adults with pathogenic AUTS2 variants, including a two-base pair deletion, further delineate the AUTS2 syndrome. European Journal of Human Genetics 23, 803-807.

Beunders, G., Kamp, J. van de, Vasudevan, P., Morton, J., Smets, K., Kleefstra, T., Munnik, S.A. de, Schuurs-Hoeijmakers, J., Ceulemans, B., Zollino, M., et al. (2016). A detailed clinical analysis of 13 patients with AUTS2 syndrome further delineates the phenotypic spectrum and underscores the behavioural phenotype. Journal of Medical Genetics 53, 523-532.

Bonasio, R., Tu, S., and Reinberg, D. (2010). Molecular Signals of Epigenetic States. Science 330, 612-616.

Briggs, J.A., Li, V.C., Lee, S., Woolf, C.J., Klein, A., and Kirschner, M.W. (2017). Mouse embryonic stem cells can differentiate via multiple paths to the same state. ELife 6, e26945.

Butler, A., Hoffman, P., Smibert, P., Papalexi, E., and Satija, R. (2018). Integrating single-cell transcriptomic data across different conditions, technologies, and species. Nature Biotechnology 36, 411-420.

Cantani, A., and Gagliesi, D. (1998). Rubinstein-Taybi syndrome. Review of 732 cases and analysis of the typical traits. Eur Rev Med Pharmacol Sci 2, 81-87.

Chang, W.-T., Chen, H., Chiou, R.-J., Chen, C.-Y., and Huang, A.-M. (2005). A novel function of transcription factor $\alpha$-Pal/NRF-1: Increasing neurite outgrowth. Biochemical and Biophysical Research Communications 334, 199-206.

Cohen, I., Zhao, D., Bar, C., Valdes, V.J., Dauber-Decker, K.L., Nguyen, M.B., Nakayama, M., Rendl, M., Bickmore, W.A., Koseki, H., et al. (2018). PRC1 Fine-tunes Gene Repression and Activation to Safeguard Skin Development and Stem Cell Specification. Cell Stem Cell 22, 726739.e7.

Di Croce, L., and Helin, K. (2013). Transcriptional regulation by Polycomb group proteins. Nat Struct Mol Biol 20, 1147-1155.

Dobin, A., Davis, C.A., Schlesinger, F., Drenkow, J., Zaleski, C., Jha, S., Batut, P., Chaisson, M., and Gingeras, T.R. (2013). STAR: ultrafast universal RNA-seq aligner. Bioinformatics 29, 15-21.

Edwards, T.J., Sherr, E.H., Barkovich, A.J., and Richards, L.J. (2014). Clinical, genetic and imaging findings identify new causes for corpus callosum development syndromes. Brain 137, 15791613.

Elia, J., Gai, X., Xie, H.M., Perin, J.C., Geiger, E., Glessner, J.T., D’arcy, M., deBerardinis, R., Frackelton, E., Kim, C., et al. (2010). Rare structural variants found in attention-deficit hyperactivity disorder are preferentially associated with neurodevelopmental genes. Molecular Psychiatry 15, 637-646. 
Endoh, M., Endo, T.A., Endoh, T., Fujimura, Y., Ohara, O., Toyoda, T., Otte, A.P., Okano, M., Brockdorff, N., Vidal, M., et al. (2008). Polycomb group proteins Ring1A/B are functionally linked to the core transcriptional regulatory circuitry to maintain ES cell identity. Development $135,1513-1524$.

Escobar, T.M., Oksuz, O., Saldaña-Meyer, R., Descostes, N., Bonasio, R., and Reinberg, D. (2019). Active and Repressed Chromatin Domains Exhibit Distinct Nucleosome Segregation during DNA Replication. Cell 179, 953-963.e11.

Escobar, T.M., Loyola, A., and Reinberg, D. (2021). Parental nucleosome segregation and the inheritance of cellular identity. Nature Reviews Genetics 1-14.

Evans, M.J., and Scarpulla, R.C. (1990). NRF-1: a trans-activator of nuclear-encoded respiratory genes in animal cells. Genes Dev 4, 1023-1034.

Francis, N.J., Kingston, R.E., and Woodcock, C.L. (2004). Chromatin Compaction by a Polycomb Group Protein Complex. Science 306, 1574-1577.

Gao, Z., Zhang, J., Bonasio, R., Strino, F., Sawai, A., Parisi, F., Kluger, Y., and Reinberg, D. (2012). PCGF Homologs, CBX Proteins, and RYBP Define Functionally Distinct PRC1 Family Complexes. Molecular Cell 45, 344-356.

Gao, Z., Lee, P., Stafford, J.M., von Schimmelmann, M., Schaefer, A., and Reinberg, D. (2014). An AUTS2-Polycomb complex activates gene expression in the CNS. Nature 516, 349-354.

Gleyzer, N., Vercauteren, K., and Scarpulla, R.C. (2005). Control of Mitochondrial Transcription Specificity Factors (TFB1M and TFB2M) by Nuclear Respiratory Factors (NRF-1 and NRF-2) and PGC-1 Family Coactivators. Mol Cell Biol 25, 1354-1366.

Gu, Z., Eils, R., and Schlesner, M. (2016). Complex heatmaps reveal patterns and correlations in multidimensional genomic data. Bioinformatics 32, 2847-2849.

Guerreiro, R., Brás, J., and Hardy, J. (2015). SnapShot: Genetics of ALS and FTD. Cell 160, 798798.e1.

Gugneja, S., and Scarpulla, R.C. (1997). Serine Phosphorylation within a Concise Amino-terminal Domain in Nuclear Respiratory Factor 1 Enhances DNA Binding*. Journal of Biological Chemistry 272, 18732-18739.

Hagemann-Jensen, M. (2020). Smart-seq3 Protocol.

Hagemann-Jensen, M., Ziegenhain, C., Chen, P., Ramsköld, D., Hendriks, G.-J., Larsson, A.J.M., Faridani, O.R., and Sandberg, R. (2020). Single-cell RNA counting at allele and isoform resolution using Smart-seq3. Nature Biotechnology 38, 708-714. 
Hattori, E., Toyota, T., Ishitsuka, Y., Iwayama, Y., Yamada, K., Ujike, H., Morita, Y., Kodama, M., Nakata, K., Minabe, Y., et al. (2009). Preliminary genome-wide association study of bipolar disorder in the Japanese population. American Journal of Medical Genetics Part B: Neuropsychiatric Genetics 150B, 1110-1117.

Hauri, S., Comoglio, F., Seimiya, M., Gerstung, M., Glatter, T., Hansen, K., Aebersold, R., Paro, R., Gstaiger, M., and Beisel, C. (2016). A High-Density Map for Navigating the Human Polycomb Complexome. Cell Reports 17, 583-595.

Heinz, S., Benner, C., Spann, N., Bertolino, E., Lin, Y.C., Laslo, P., Cheng, J.X., Murre, C., Singh, H., and Glass, C.K. (2010). Simple Combinations of Lineage-Determining Transcription Factors Prime cis-Regulatory Elements Required for Macrophage and B Cell Identities. Molecular Cell 38, 576-589.

Hori, K., and Hoshino, M. (2017). Neuronal Migration and AUTS2 Syndrome. Brain Sciences 7, 54.

Hori, K., Nagai, T., Shan, W., Sakamoto, A., Taya, S., Hashimoto, R., Hayashi, T., Abe, M., Yamazaki, M., Nakao, K., et al. (2014). Cytoskeletal Regulation by AUTS2 in Neuronal Migration and Neuritogenesis. Cell Reports 9, 2166-2179.

Hsiao, H.-Y., Jukam, D., Johnston, R., and Desplan, C. (2013). The neuronal transcription factor erect wing regulates specification and maintenance of Drosophila R8 photoreceptor subtypes. Developmental Biology 381, 482-490.

Huo, L., and Scarpulla, R.C. (2001). Mitochondrial DNA Instability and Peri-Implantation Lethality Associated with Targeted Disruption of Nuclear Respiratory Factor 1 in Mice. Molecular and Cellular Biology 21, 644-654.

Jordan, V.K., Fregeau, B., Ge, X., Giordano, J., Wapner, R.J., Balci, T.B., Carter, M.T., Bernat, J.A., Moccia, A.N., Srivastava, A., et al. (2018). Genotype-phenotype correlations in individuals with pathogenic RERE variants. Human Mutation 39, 666-675.

Kapoor, M., Wang, J.-C., Wetherill, L., Le, N., Bertelsen, S., Hinrichs, A.L., Budde, J., Agrawal, A., Bucholz, K., Dick, D., et al. (2013). A meta-analysis of two genome-wide association studies to identify novel loci for maximum number of alcoholic drinks. Hum Genet 132, 1141-1151.

Kasinath, V., Beck, C., Sauer, P., Poepsel, S., Kosmatka, J., Faini, M., Toso, D., Aebersold, R., and Nogales, E. (2021). JARID2 and AEBP2 regulate PRC2 in the presence of H2AK119ub1 and other histone modifications. Science 371.

Kim, J., and Kingston, R.E. (2020). The CBX family of proteins in transcriptional repression and memory. J Biosci 45, 16. 
Kiyama, T., Chen, C.-K., Wang, S.W., Pan, P., Ju, Z., Wang, J., Takada, S., Klein, W.H., and Mao, C.-A. (2018). Essential roles of mitochondrial biogenesis regulator Nrf1 in retinal development and homeostasis. Molecular Neurodegeneration 13, 56.

Kiyama, T., Long, Y., Chen, C.-K., Whitaker, C.M., Shay, A., Wu, H., Badea, T.C., Mohsenin, A., Parker-Thornburg, J., Klein, W.H., et al. (2019). Essential Roles of Tbr1 in the Formation and Maintenance of the Orientation-Selective J-RGCs and a Group of OFF-Sustained RGCs in Mouse. Cell Reports 27, 900-915.e5.

Kloet, S.L., Makowski, M.M., Baymaz, H.I., van Voorthuijsen, L., Karemaker, I.D., Santanach, A., Jansen, P.W.T.C., Di Croce, L., and Vermeulen, M. (2016). The dynamic interactome and genomic targets of Polycomb complexes during stem cell differentiation. Nat Struct Mol Biol 23, 682-690.

Korotkevich, G., Sukhov, V., and Sergushichev, A. (2019). Fast gene set enrichment analysis. BioRxiv 060012.

Korsunsky, I., Nathan, A., Millard, N., and Raychaudhuri, S. (2019). Presto scales Wilcoxon and auROC analyses to millions of observations. BioRxiv 653253.

Langmead, B., and Salzberg, S.L. (2012). Fast gapped-read alignment with Bowtie 2. Nat Methods 9, 357-359.

Lau, M.S., Schwartz, M.G., Kundu, S., Savol, A.J., Wang, P.I., Marr, S.K., Grau, D.J., Schorderet, P., Sadreyev, R.I., Tabin, C.J., et al. (2017). Mutation of a nucleosome compaction region disrupts Polycomb-mediated axial patterning. Science 355, 1081-1084.

LeRoy, G., Oksuz, O., Descostes, N., Aoi, Y., Ganai, R.A., Kara, H.O., Yu, J.-R., Lee, C.-H., Stafford, J., Shilatifard, A., et al. (2019). LEDGF and HDGF2 relieve the nucleosome-induced barrier to transcription in differentiated cells. Science Advances 5, eaay3068.

Li, G., Margueron, R., Ku, M., Chambon, P., Bernstein, B.E., and Reinberg, D. (2010). Jarid2 and PRC2, partners in regulating gene expression. Genes Dev 24, 368-380.

Li, H., Handsaker, B., Wysoker, A., Fennell, T., Ruan, J., Homer, N., Marth, G., Abecasis, G., Durbin, R., and Subgroup, 1000 Genome Project Data Processing (2009). The Sequence Alignment/Map format and SAMtools. Bioinformatics 25, 2078-2079.

Liao, Y., Smyth, G.K., and Shi, W. (2014). featureCounts: an efficient general purpose program for assigning sequence reads to genomic features. Bioinformatics 30, 923-930.

Loubiere, V., Papadopoulos, G.L., Szabo, Q., Martinez, A.-M., and Cavalli, G. (2020). Widespread activation of developmental gene expression characterized by PRC1-dependent chromatin looping. Science Advances 6, eaax4001. 
Margueron, R., and Reinberg, D. (2011). The Polycomb complex PRC2 and its mark in life. Nature 469, 343-349.

Martinez-Delgado, B., Lopez-Martin, E., Lara-Herguedas, J., Monzon, S., Cuesta, I., Juliá, M., Aquino, V., Rodriguez-Martin, C., Damian, A., Gonzalo, I., et al. (2020). De novo small deletion affecting transcription start site of short isoform of AUTS2 gene in a patient with syndromic neurodevelopmental defects. Am J Med Genet A.

Mazzoni, E.O., Mahony, S., Peljto, M., Patel, T., Thornton, S.R., McCuine, S., Reeder, C., Boyer, L.A., Young, R.A., Gifford, D.K., et al. (2013). Saltatory remodeling of Hox chromatin in response to rostrocaudal patterning signals. Nature Neuroscience 16, 1191-1198.

Mefford, H.C., Muhle, H., Ostertag, P., Spiczak, S. von, Buysse, K., Baker, C., Franke, A., Malafosse, A., Genton, P., Thomas, P., et al. (2010). Genome-Wide Copy Number Variation in Epilepsy: Novel Susceptibility Loci in Idiopathic Generalized and Focal Epilepsies. PLOS Genetics 6, e1000962.

Min, J., Zhang, Y., and Xu, R.-M. (2003). Structural basis for specific binding of Polycomb chromodomain to histone H3 methylated at Lys 27. Genes Dev. 17, 1823-1828.

Narendra, V., Rocha, P.P., An, D., Raviram, R., Skok, J.A., Mazzoni, E.O., and Reinberg, D. (2015). CTCF establishes discrete functional chromatin domains at the Hox clusters during differentiation. Science 347, 1017-1021.

Ogryzko, V.V., Schiltz, R.L., Russanova, V., Howard, B.H., and Nakatani, Y. (1996). The Transcriptional Coactivators p300 and CBP Are Histone Acetyltransferases. Cell 87, 953-959.

Oksenberg, N., and Ahituv, N. (2013). The role of AUTS2 in neurodevelopment and human evolution. Trends in Genetics 29, 600-608.

Oksenberg, N., Haliburton, G.D.E., Eckalbar, W.L., Oren, I., Nishizaki, S., Murphy, K., Pollard, K.S., Birnbaum, R.Y., and Ahituv, N. (2014). Genome-wide distribution of Auts2 binding localizes with active neurodevelopmental genes. Translational Psychiatry 4, e431-e431.

Oksuz, O., Narendra, V., Lee, C.-H., Descostes, N., LeRoy, G., Raviram, R., Blumenberg, L., Karch, K., Rocha, P.P., Garcia, B.A., et al. (2018). Capturing the Onset of PRC2-Mediated Repressive Domain Formation. Molecular Cell 70, 1149-1162.e5.

Orlando, D.A., Chen, M.W., Brown, V.E., Solanki, S., Choi, Y.J., Olson, E.R., Fritz, C.C., Bradner, J.E., and Guenther, M.G. (2014). Quantitative ChIP-Seq Normalization Reveals Global Modulation of the Epigenome. Cell Reports 9, 1163-1170.

Palmer, E.E., Hong, S., Al Zahrani, F., Hashem, M.O., Aleisa, F.A., Ahmed, H.M.J., Kandula, T., Macintosh, R., Minoche, A.E., Puttick, C., et al. (2019). De Novo Variants Disrupting the HX Repeat Motif of ATN1 Cause a Recognizable Non-Progressive Neurocognitive Syndrome. The American Journal of Human Genetics 104, 542-552. 
Parekh, S., Ziegenhain, C., Vieth, B., Enard, W., and Hellmann, I. (2018). zUMIs - A fast and flexible pipeline to process RNA sequencing data with UMIs. Gigascience 7.

Pasini, D., Cloos, P.A.C., Walfridsson, J., Olsson, L., Bukowski, J.-P., Johansen, J.V., Bak, M., Tommerup, N., Rappsilber, J., and Helin, K. (2010). JARID2 regulates binding of the Polycomb repressive complex 2 to target genes in ES cells. Nature 464, 306-310.

Quinlan, A.R., and Hall, I.M. (2010). BEDTools: a flexible suite of utilities for comparing genomic features. Bioinformatics 26, 841-842.

Ramírez, F., Ryan, D.P., Grüning, B., Bhardwaj, V., Kilpert, F., Richter, A.S., Heyne, S., Dündar, F., and Manke, T. (2016). deepTools2: a next generation web server for deep-sequencing data analysis. Nucleic Acids Res 44, W160-W165.

Reyes, N.S.D.L., Bragg-Gonzalo, L., and Nieto, M. (2020). Development and plasticity of the corpus callosum. Development 147.

RUBINSTEIN, J.H., and TAYBI, H. (1963). Broad thumbs and toes and facial abnormalities. A possible mental retardation syndrome. Am J Dis Child 105, 588-608.

Scarpulla, R.C. (2011). Metabolic control of mitochondrial biogenesis through the PGC-1 family regulatory network. Biochim Biophys Acta 1813, 1269-1278.

Scelfo, A., Fernández-Pérez, D., Tamburri, S., Zanotti, M., Lavarone, E., Soldi, M., Bonaldi, T., Ferrari, K.J., and Pasini, D. (2019). Functional Landscape of PCGF Proteins Reveals Both RING1A/B-Dependent-and RING1A/B-Independent-Specific Activities. Molecular Cell 74, 10371052.e7.

Schuettengruber, B., Bourbon, H.-M., Di Croce, L., and Cavalli, G. (2017). Genome Regulation by Polycomb and Trithorax: 70 Years and Counting. Cell 171, 34-57.

Sobreira, N., Schiettecatte, F., Valle, D., and Hamosh, A. (2015). GeneMatcher: A Matching Tool for Connecting Investigators with an Interest in the Same Gene. Human Mutation 36, 928-930.

Stevens, C.A. (1993). Rubinstein-Taybi Syndrome. In GeneReviews ${ }^{\circledR}$, M.P. Adam, H.H. Ardinger, R.A. Pagon, S.E. Wallace, L.J. Bean, K. Stephens, and A. Amemiya, eds. (Seattle (WA): University of Washington, Seattle), p.

Subramanian, A., Tamayo, P., Mootha, V.K., Mukherjee, S., Ebert, B.L., Gillette, M.A., Paulovich, A., Pomeroy, S.L., Golub, T.R., Lander, E.S., et al. (2005). Gene set enrichment analysis: A knowledge-based approach for interpreting genome-wide expression profiles. Proceedings of the National Academy of Sciences 102, 15545-15550.

Sultana, R., Yu, C.-E., Yu, J., Munson, J., Chen, D., Hua, W., Estes, A., Cortes, F., de la Barra, F., Yu, D., et al. (2002). Identification of a Novel Gene on Chromosome 7q11.2 Interrupted by a Translocation Breakpoint in a Pair of Autistic Twins. Genomics 80, 129-134. 
Tavares, L., Dimitrova, E., Oxley, D., Webster, J., Poot, R., Demmers, J., Bezstarosti, K., Taylor, S., Ura, H., Koide, H., et al. (2012). RYBP-PRC1 Complexes Mediate H2A Ubiquitylation at Polycomb Target Sites Independently of PRC2 and H3K27me3. Cell 148, 664-678.

Tong, C.-W., Wang, J.-L., Jiang, M.-S., Hsu, C.-H., Chang, W.-T., and Huang, A.-M. (2013). Novel genes that mediate nuclear respiratory factor 1-regualted neurite outgrowth in neuroblastoma IMR-32 cells. Gene 515, 62-70.

Trojer, P., and Reinberg, D. (2007). Facultative Heterochromatin: Is There a Distinctive Molecular Signature? Molecular Cell 28, 1-13.

Ufartes, R., Berger, H., Till, K., Salinas, G., Sturm, M., Altmüller, J., Nürnberg, P., Thiele, H., Funke, R., Apeshiotis, N., et al. (2020). De novo mutations in FBRSL1 cause a novel recognizable malformation and intellectual disability syndrome. Hum Genet 139, 1363-1379.

Voigt, P., Tee, W.-W., and Reinberg, D. (2013). A double take on bivalent promoters. Genes Dev. $27,1318-1338$.

Wang, R., Taylor, A.B., Leal, B.Z., Chadwell, L.V., Ilangovan, U., Robinson, A.K., Schirf, V., Hart, P.J., Lafer, E.M., Demeler, B., et al. (2010). Polycomb Group Targeting through Different Binding Partners of RING1B C-Terminal Domain. Structure 18, 966-975.

Wichterle, H., Lieberam, I., Porter, J.A., and Jessell, T.M. (2002). Directed Differentiation of Embryonic Stem Cells into Motor Neurons. Cell 110, 385-397.

Wiley, S., Swayne, S., Rubinstein, J.H., Lanphear, N.E., and Stevens, C.A. (2003). RubinsteinTaybi syndrome medical guidelines. American Journal of Medical Genetics Part A 119A, 101110.

Yu, G., Wang, L.-G., and He, Q.-Y. (2015). ChIPseeker: an R/Bioconductor package for ChIP peak annotation, comparison and visualization. Bioinformatics 31, 2382-2383.

Yu, J.-R., Lee, C.-H., Oksuz, O., Stafford, J.M., and Reinberg, D. (2019). PRC2 is high maintenance. Genes Dev. 33, 903-935.

Zha, X., Costa, V., Harding, A.M.S., Reznikov, L., Benson, C.J., and Welsh, M.J. (2009). ASIC2 Subunits Target Acid-Sensing Ion Channels to the Synapse via an Association with PSD-95. J. Neurosci. 29, 8438-8446.

Zhang, Y., Liu, T., Meyer, C.A., Eeckhoute, J., Johnson, D.S., Bernstein, B.E., Nusbaum, C., Myers, R.M., Brown, M., Li, W., et al. (2008). Model-based Analysis of ChIP-Seq (MACS). Genome Biology 9, R137. 